\title{
THE PROPER MOTION OF THE ARCHES CLUSTER WITH KECK LASER-GUIDE STAR ADAPTIVE OPTICS
}

\author{
A. Stolte, ${ }^{1}$ A. M. Ghez, ${ }^{1,2}$ M. Morris, ${ }^{1}$ J. R. Lu, ${ }^{1}$ W. Brandner, ${ }^{1,3}$ and K. Matthews ${ }^{4}$ \\ Received 2007 May 1; accepted 2007 November 10
}

\begin{abstract}
We present the first measurement of the proper motion of the young, compact Arches cluster near the Galactic center from near-infrared adaptive optics (AO) data taken with the recently commissioned laser-guide star (LGS) at the Keck $10 \mathrm{~m}$ telescope. The excellent astrometric accuracy achieved with LGS-AO provides the basis for a detailed comparison with VLT/NAOS-CONICA data taken 4.3 yr earlier. Over the 4.3 yr baseline, a spatial displacement of the Arches cluster with respect to the field population is measured to be $24.0 \pm 2.2 \mathrm{mas}$, corresponding to a proper motion of $5.6 \pm 0.5 \mathrm{mas} \mathrm{yr}^{-1}$ or $212 \pm 29 \mathrm{~km} \mathrm{~s}^{-1}$ at a distance of $8 \mathrm{kpc}$. In combination with the known line-of-sight velocity of the cluster, we derive a three-dimensional (3D) space motion of $232 \pm 30 \mathrm{~km} \mathrm{~s}^{-1}$ of the Arches relative to the field. The large proper motion of the Arches cannot be explained with any of the closed orbital families observed in gas clouds in the bar potential of the inner Galaxy, but would be consistent with the Arches being on a transitional trajectory between $\mathrm{x} 1$ and $\mathrm{x} 2$ orbits. We investigate a cloud-cloud collision as the possible origin for the Arches cluster. The integration of the cluster orbit in the potential of the inner Galaxy suggests that the cluster passes within $10 \mathrm{pc}$ of the supermassive black hole only if its true GC distance is very close to its projected distance. A contribution of young stars from the Arches cluster to the young stellar population in the inner few parsecs of the GC thus appears increasingly unlikely. The measurement of the $3 \mathrm{D}$ velocity and orbital analysis provides the first observational evidence that Arches-like clusters do not spiral into the GC. This confirms that no progenitor clusters to the nuclear cluster are observed at the present epoch.
\end{abstract}

Subject headings: astrometry - Galaxy: center — open clusters and associations: individual (Arches) techniques: high angular resolution

Online material: color figures

\section{INTRODUCTION}

The Arches cluster is among the very few massive starburst clusters observed in the inner Milky Way. At projected distances below $30 \mathrm{pc}$ from the Galactic center (GC), only two other dense, young clusters are known: the Quintuplet and the central cluster. Obtaining an unbiased sample of cluster members and thus an estimate of the stellar mass function is difficult for the latter two clusters; the central cluster contains a mixed variety of stars having different ages, and the 4 Myr "old" Quintuplet cluster appears already widely dispersed (see, e.g., Fig. 2 in Figer et al. 1999). The compactness of the Arches cluster and its young, uniform age of only $\sim 2 \mathrm{Myr}$ (Najarro et al. 2004; Figer et al. 2002) characterize this cluster as a unique target to study the stellar mass function and dynamical properties of clusters forming in the immediate neighborhood of the center of our Galaxy.

The inner $\sim 100 \mathrm{pc}$ of the nuclear region of the Galaxy are a hostile environment for star cluster survival. Clusters like the Arches and Quintuplet are expected to disrupt in the intense tidal field on a timescale of $\sim 10 \mathrm{Myr}$ (Kim et al. 1999; Portegies Zwart et al. 2002). The strong influence of external tidal forces on the evolution of these clusters led to the suggestion by Gerhard (2001) that massive, young clusters migrate inwards from tens of parsecs to the inner few parsecs around the black hole. Gerhard suggested that the cores of these clusters might survive tidal dis-

\footnotetext{
${ }^{1}$ Division of Astronomy and Astrophysics, UCLA, Los Angeles, CA 900951547, astolte@astro.ucla.edu, ghez@astro.ucla.edu,morris@astro.ucla.edu, jlu@ astro.ucla.edu.

2 Institute of Geophysics and Planetary Physics, UCLA, Los Angeles, CA 90095.

3 Max-Planck-Institut for Astronomy, Königstuhl 17, 69117 Heidelberg, Germany, brandner@astro.ucla.edu.

${ }^{4}$ Caltech Optical Observatories, California Institute of Technology, MS 320-47, Pasadena, CA91225,kym@caltech.edu.
}

ruption and supply the population of young, high-mass stars observed close to the black hole, where conditions are inhospitable to in situ star formation (Morris 1993; Ghez et al. 2003, 2005). However, dynamical simulations suggest that a cluster mass of $M_{\mathrm{cl}}>10^{6} M_{\odot}$ is required for a dense core to reach the central parsec within the lifetime of the high-mass stars (Kim \& Morris 2003), much more massive than the Arches today with $M_{\mathrm{cl}} \sim 10^{4} M_{\odot}$.

The amount of material that was already stripped from the cluster and thus the initial cluster mass depend on its orbit in the GC potential and the true distance of the cluster to the GC. At its projected distance of $26 \mathrm{pc}$, the Arches could have lost $50 \%$ of its initial mass, such that the cluster could have been as massive as $2 \times 10^{4} M_{\odot}$ initially ( Kim et al. 2000). Thus far, both the orbit and the true distance to the GC, $r_{\mathrm{GC}}$, are observationally unconstrained. Kim et al. (2000) and Portegies Zwart et al. (2002) attempted to estimate $r_{\mathrm{GC}}$ from $N$-body simulations of Arches-like clusters moving on circular orbits in a spherically symmetric potential representing the $\mathrm{GC}$ tidal field. Both studies compared the surface density profiles of dynamically evolving clusters to the surface density of massive stars observed in the Arches. Despite similar initial conditions (cluster mass, age, Galactic potential), the most likely GC distance ranges from $30-50 \mathrm{pc}$, favoring $30 \mathrm{pc}$ (Kim et al. 2000), to 50-90 pc, favoring 90 pc (Portegies Zwart et al. 2002). The measurement of the three-dimensional space motion of the Arches would provide a strong observational constraint on the cluster orbit. An estimate of the true distance from the GC and of the orbital timescale limits the amount of material stripped during the cluster's lifetime, and thus constrains the initial mass of the Arches and the galactocentric radius where the complete tidal dissolution of the cluster is expected.

In this paper we present an astrometric comparison of new laser guide star adaptive optics (LGS-AO) observations obtained 
at Keck with natural guide star (NGS) AO data observed $4.3 \mathrm{yr}$ earlier with the Very Large Telescope (VLT). The high spatial resolution and astrometric accuracy of the AO observations allow us to derive the proper motion of the Arches cluster with respect to the field population. In combination with the known line-of-sight velocity, the 3D space motion of the cluster provides the first observational constraints on the cluster orbit.

We present our observations, data reduction and photometric calibration in $\S 2$. The critical step of astrometric matching between the NIRC2 and NACO data sets is explained in $\S 2.3$. The proper motion is derived in $\S 3$, and a discussion of the $3 \mathrm{D}$ space motion of the cluster is presented in $\S 4$. The $3 \mathrm{D}$ orbit of the cluster in the GC potential is modeled in $\S 5$, followed by our conclusions in $\S 6$.

\section{OBSERVATIONS}

\subsection{Keck/NIRC2}

Keck II LGS-AO observations were obtained on 2006 July 18. The NIRC2 narrow camera with a pixel scale of 10 mas pixel ${ }^{-1}$ $\left(0.01^{\prime \prime}\right.$ pixel $\left.^{-1}\right)$ was employed to obtain a set of 50 images of the Arches cluster in $K^{\prime}\left(\lambda_{c}=2.12 \mu \mathrm{m}, \Delta \lambda=0.35 \mu \mathrm{m}\right)$. The laser guide probe provided a guide source with $V \sim 10 \mathrm{mag}$ brightness centered in the field of view of the NIRC2 camera. A foreground giant with $R=15.2$ mag located $10^{\prime \prime}$ from the field center was used as the tip-tilt reference source. The 5 mag brighter LGS-as opposed to the faint tip-tilt reference source previously available as a natural guide star (NGS)-delivered images very close to the diffraction limit of 52 mas at $2.12 \mu \mathrm{m}$. These observations exceed the resolution obtained with earlier NGS observations (Stolte et al. 2005) by a factor of 1.5 , which facilitates resolving the stellar population in the crowding limited cluster field. To aid reconstruction of bad pixels in NIRC2 and avoid detector persistence from bright sources, the telescope was dithered in a random dither pattern with three images taken at each position, with individual integration times of $30 \mathrm{~s}$ per frame, split into 10 co-adds $\times 3 \mathrm{~s}$ in the $K^{\prime}$ filter to avoid saturation of most bright sources. The visual seeing during these observations was $0.8^{\prime \prime}-1.2^{\prime \prime}$, which is worse than average for Mauna Kea conditions, leading to larger variations than expected in AO performance. Thirty-six $K^{\prime}$-band frames displaying the best AO performance were selected, leading to a total exposure time of 18 minutes in $K^{\prime}$. These data form the basis of the proper motion study.

In addition, 15 images in $H\left(\lambda_{c}=1.63 \mu \mathrm{m}, \Delta \lambda=0.30 \mu \mathrm{m}\right)$ were obtained on 2006 May 21 with the same observational setup. The fainter source brightness in $H$ allowed for longer integration times of $10 \mathrm{~s} \times 3 \mathrm{co-adds}$, and a box five dither pattern was used with three images taken at each position. Varying AO performance led to the selection of the nine images displaying core FWHM $<65$ mas, with a total exposure time of 4.5 minutes. The $H$-band data are used supplementary to the $K^{\prime}$ images to reject artifacts from the final catalog.

The standard imaging reduction steps including flat fielding, sky and dark subtraction, and removal of cosmic-ray events are detailed below. The science frames were reduced using flat fields and offset sky frames taken during the same night. Individual bad pixel masks were created from a combination of dark, flat-field, and cosmic ray masking of individual exposures. Strehl ratios on preselected $K^{\prime}$ frames ranged from 0.25 to 0.44 (median 0.34 ) corresponding to a point-spread function (PSF) core FWHM between 52 and 63 mas (median 56 mas). $H$-band Strehls covered $0.15-0.19$ (median 0.18 ) with a PSF core FWHM of 58-65 mas (median 60 mas). In order to enhance the resolution in the final image, each frame was weighted by the Strehl ratio prior to im- age combination. The individual bad pixel mask was applied to each frame, and frames were averaged together using the drizzle algorithm (Fruchter \& Hook 2002). The combined images display a core FWHM of 53 mas in $K^{\prime}$ and 60 mas in $H$. In addition to the deep $K^{\prime}$ frame, three auxiliary $K^{\prime}$ frames were formed by co-adding 12 individual images in each frame. These auxiliary frames were used for a first pass rejection of image and PSF artifacts, as well as for uncertainty estimates.

Stellar positions and photometry were extracted from the final, averaged NIRC2 images using the Starfinder code (Diolaiti et al. 2000). This code has the advantage of constructing the PSF from the image empirically, without the need to assume an analytic function. This is particularly critical for the complex AO PSFs displaying steep kernels with structured halos and Airy ring patterns. In the crowded stellar field of the Arches, 10 stars were used to create the PSF. The detection threshold was set to $3 \sigma$ above the local background, and a PSF correlation threshold of 0.7 was imposed. The relatively low correlation threshold enables us to include close neighbors in the final photometry file, while higher thresholds tend to omit faint sources embedded in the halos of bright stars. In order to reject false detections, each star entering the calibrated $K^{\prime}$ catalog was required to be detected in the deep $K^{\prime}$ exposure and in all three auxiliary frames. This strict requirement led to the rejection of most PSF and image artifacts. Any remaining PSF artifacts were removed during the final catalog matching. The calibrated NIRC $2 K^{\prime}$ catalog contained 869 objects in the magnitude range $10.3<K^{\prime}<20.5$ mag on the combined $10.8^{\prime \prime} \times 10.8^{\prime \prime}$ image. Photometric calibration was performed by zero-pointing against the NACO photometry presented in Stolte et al. (2005), and the comparison of $\left(H-K^{\prime}\right)_{\mathrm{NIRC} 2}$ with $\left(H-K_{s}\right)_{\text {NACO }}$ yielded no color terms. A detailed photometric analysis of the cluster population will follow in a subsequent paper.

\subsection{VLT/NAOS-CONICA}

The Keck/NIRC2 $K^{\prime}$ astrometric data set was combined with VLT/NACO data taken on 2002 March 31, providing a baseline of $4.3 \mathrm{yr}$ for the proper motion study. NACO $H$ and $K_{s}$ images with a total integration time of 14 and 7 minutes, respectively, were obtained with the medium camera with a plate scale of 27 mas pixel $^{-1}$. The NACO data were taken with a natural guide star (NGS) on the NAOS optical wavefront sensor. Because of the faintness of the NGS in the Arches field $(R=15.2 \mathrm{mag})$, the Strehl ratios achieved were 0.20 in $K_{s}$ and 0.14 in $H$, providing a FWHM resolution of 84 mas in both filters. The merged catalog of sources detected in both $H$ and $K_{s}$ contains 487 stars in the magnitude range $10.2<K_{s}<20.7 \mathrm{mag}$, and provides the basis for the proper motion measurement. A comparison between the NIRC2 $K^{\prime}$ and NACO $K_{s}$ images is shown in Figure 1. For a description of the NACO data and analysis see Stolte et al. (2005).

\subsection{Geometric Transformation and Astrometry}

With the aim of measuring the relative motion of stars in the Arches field, the spatial displacement of each star over the $4.3 \mathrm{yr}$ baseline is calculated. Bright sources on the combined NIRC2 $K^{\prime}$ image provide the reference frame for the proper motion analysis. A geometric transformation to convert NACO pixel positions to the NIRC2 frame was derived using the IRAF geomap task. By deriving the transformation from bright and intermediate sources measured directly on both images, the different optical distortions between NIRC2 and NACO are taken into account within the limitations of the two-dimensional, second-order polynomial fit performed with geomap. The geometric solution is created iteratively 

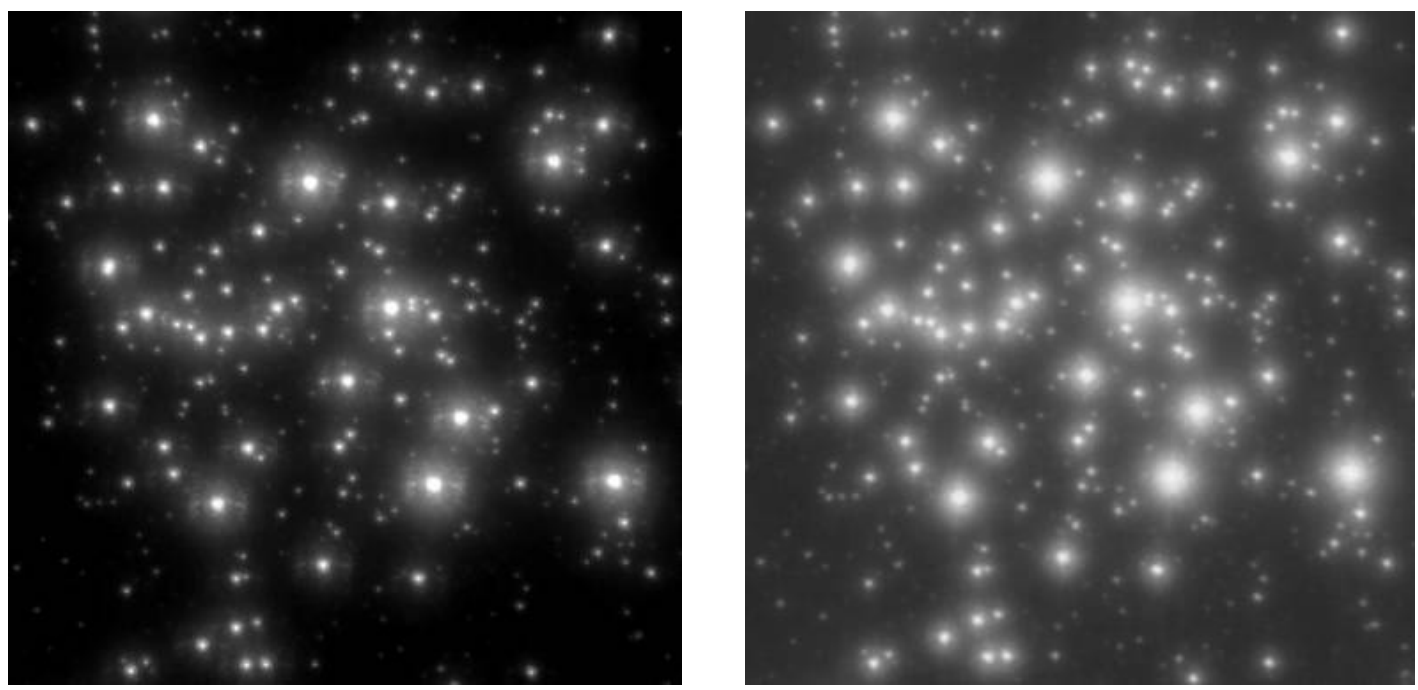

FIG. 1.-LGS-AO Keck/NIRC2 $K^{\prime}$ image (left) with a spatial resolution of 53 mas (FWHM PSF) compared to the NGS-AO VLT/NACO $K_{s}$ image with 84 mas (FWHM PSF). The Keck $10 \mathrm{~m}$ diffraction limited image displays the first-order Airy rings around each star. North is up, and east is to the left. [See the electronic edition of the Journal for a color version of this figure.]

by minimizing the $x, y$ residual displacements. The final residual rms deviation was 2 and 0.7 mas in the $x$ - and $y$-direction, respectively. In the following analysis, we therefore assume a systematic contribution of 2 mas in the proper motion uncertainty.

The selection of proper motion members was based on matching the NIRC2 source catalog with the transformed NACO catalog using a matching radius of 40 mas. This radius provided the detection of most moving sources while avoiding wrong matches with close neighbors in the dense stellar cluster field. In order to exclude artifacts from the complex PSF structure, we require that each source be detected in either the NACO or NIRC2 $H$-band image in addition to both $K$-band images. After blind matching, the matched sources were visually inspected on the NIRC2 and NACO $K$-band images, and 13 sources with merged centroids in NACO due to the lower resolution or located on the edge of the $10^{\prime \prime}$ NIRC2 field of view were removed from the matched cata$\log$, as the biased definition of their stellar centroids affected their apparent motion. The final proper motion catalog comprises 419 objects.

\section{RESULTS}

\subsection{The Relative Motion of the Arches to the Field}

The proper motion of the Arches cluster is derived from the relative motion of the cluster with respect to field stars. This relative motion depends on the geometric transformation between both data sets. As detailed below, the transformation is chosen such that proper motions over the $4.3 \mathrm{yr}$ baseline are measured in the reference frame of the cluster. Before deriving the proper motion of the cluster, this reference frame will be introduced.

The geometric transformation between NACO and NIRC 2 was derived from bright sources in the magnitude range $11<K_{s}<$ $14.5 \mathrm{mag}$. As can be seen in the color-magnitude diagram (CMD; Fig. 2), this regime is composed of stars on the Arches main sequence and thus likely cluster members. By defining the transformation from cluster stars, a cluster reference frame is created in which Arches members cluster around an east-west motion of $\Delta \mathrm{EW}=0$, and north-south motion $\Delta \mathrm{NS}=0$, implying that in the cluster reference frame the net spatial displacement of the cluster should be zero. The spatial displacement of stars in the Arches field is shown in east-west and north-south motion in Figure 3.
Two distinct features dominate in this diagram: (1) the dense clump of stars clustering around $(0,0)$, which are cluster member candidates in the cluster reference frame, and (2) the scattered population of sources in the bottom right quadrant.

The fact that Figure 3 reveals two distinct populations provides a means of identifying Arches cluster members based on proper motions. The 1 and $2 \sigma$ circles from a Gaussian fit to the relative motion distribution centered on zero are enveloping member candidates in Figure 3, and Arches members are selected with less than $2 \sigma=12.8$ mas spatial displacement in $4.3 \mathrm{yr}$, corresponding to a proper motion $\leq 3.0$ mas $\mathrm{yr}^{-1}$. While cluster candidates are confined to a dense clump with small intrinsic scatter

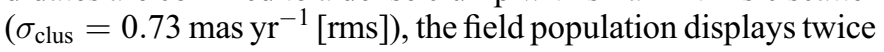

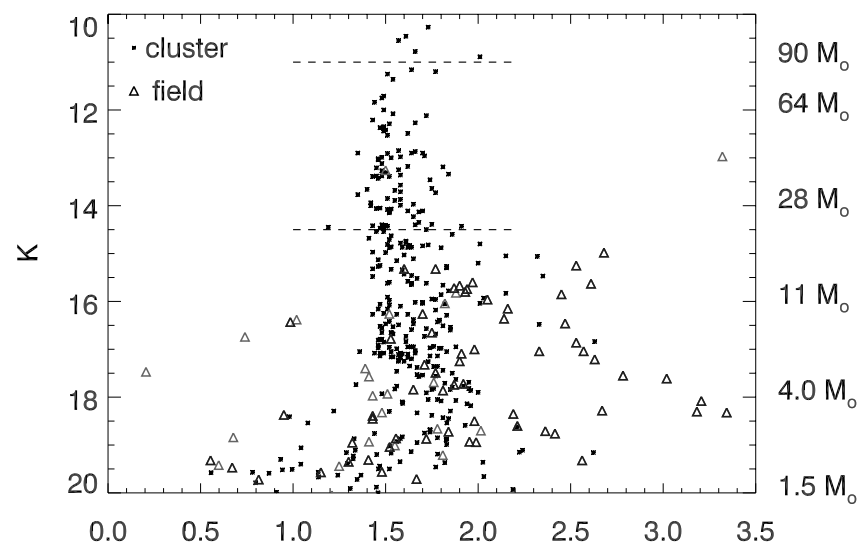

FIG. 2.-Color-magnitude diagram of the central Arches cluster $(r<0.25 \mathrm{pc})$. Proper-motion selected cluster candidates (Fig. 3, black asterisks) predominantly fill the Arches main sequence, while likely field stars (triangles) display redder colors and a larger scatter. Green triangles denote reference field stars used to derive the cluster proper motion, while red triangles are foreground candidates with irregular motions. The bright stars on the Arches main sequence (dashed lines) define the geometric transformation between the NACO and NIRC2 $\mathrm{K}$-band images. For the faintest stars, the deeper NACO $H$-band magnitudes are matched with NIRC2 $K^{\prime}$. The mismatch in resolution between the two data sets introduces additional scatter. The mass conversion for a $2 \mathrm{Myr}$ Geneva main-sequence isochrone (Lejeune \& Schaerer 2001) representing the cluster is indicated for reference on the right axis. [See the electronic edition of the Journal for a color version of this figure.] 


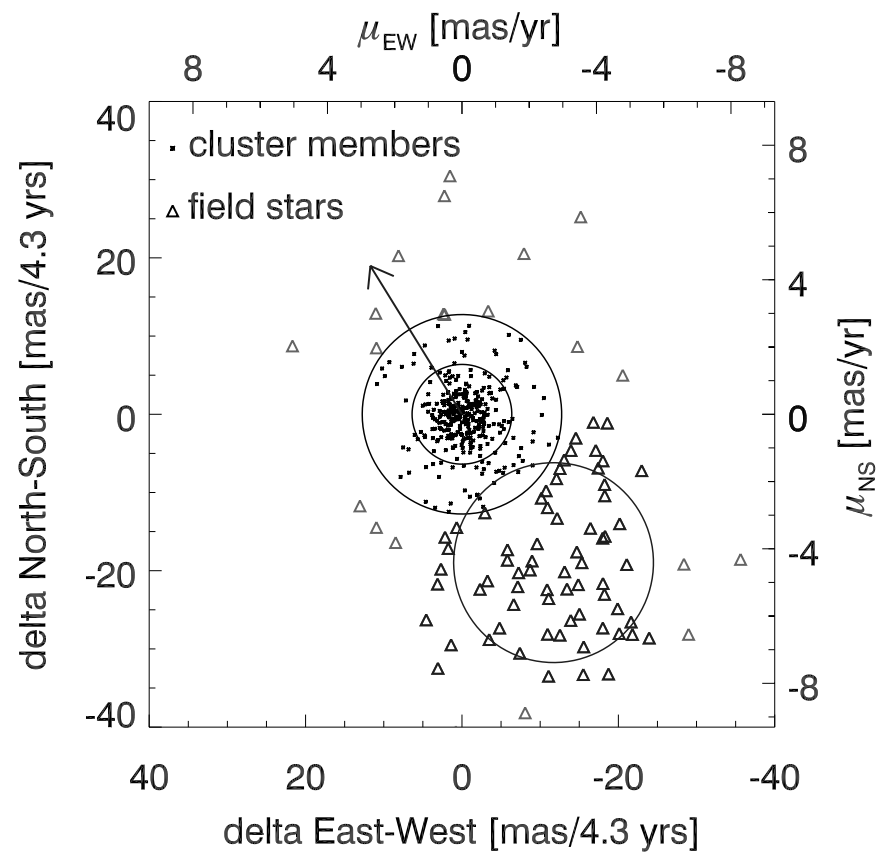

FIG. 3.- Spatial displacement in the east-west and north-south directions over $4.3 \mathrm{yr}$ in the cluster reference frame. The top and right axes display the corresponding proper motion. Arches candidate members cluster around $(0,0)($ dots $)$, while likely field stars (triangles) scatter to relative motions as large as 40 mas/ $4.3 \mathrm{yr}$ or $9.3 \mathrm{mas} \mathrm{yr}^{-1}$. The 1 and $2 \sigma$ error circles from a Gaussian fit to cluster candidate motions are shown as black circles, and stars with proper motions less than $2 \sigma$ are selected as member candidates. The bulk of the field stars are located in the southwest quadrant, indicating that the cluster moves to the northeast with respect to the field population. The mean relative velocity of field stars (green triangles) yields a cluster motion of $212 \pm 29 \mathrm{~km} \mathrm{~s}^{-1}$ to the northeast. The green circle denotes the expected proper motion regime of field stars moving on circular orbits in the bulge potential, as derived from the line-of-sight velocity boundaries in Fig. 5. Sources marked in red were excluded due to their large deviation from the mean of the field population. [See the electronic edition of the Journal for a color version of this figure.]

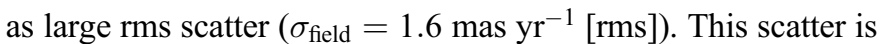
expected if we observe a mixed population of stars tangentially or radially along their orbits over a large range of distances. Field stars along the line of sight are expected to display a wide range in dynamical properties depending on their orbits. It is therefore surprising that field stars are predominantly found in the southwest quadrant in Figure 3. This apparent southwest displacement of field stars in the Arches reference frame can most easily be explained by the Arches moving to the northeast with respect to the field. The observed northeast motion of the cluster is consistent with a recent prediction from the X-ray emission of the bow shock created by the Arches during its impact on the nearby cloud (Wang et al. 2006). The median displacement of the field in the cluster reference frame is $24.0 \pm 2.2 \mathrm{mas} / 4.3 \mathrm{yr}$ or $5.6 \pm$ $0.5 \mathrm{mas} \mathrm{yr}^{-1}$, which translates to a relative motion of the cluster with respect to the field of $\mu_{\text {Arches }}=212 \pm 20 \mathrm{~km} \mathrm{~s}^{-1}$ assuming a distance of $8 \mathrm{kpc}$. With a position angle (P.A.) of $31.7^{\circ} \pm 3.8^{\circ}$ as measured from north to east, the direction of the proper motion vector is parallel to the Galactic plane (P.A. $=34.8^{\circ}$ ) within the uncertainties.

Before employing the proper motion to derive the threedimensional space motion of the cluster, we investigate the sources of uncertainty contributing to the proper motion measurement. The quoted accuracy in the proper motion measurement is achieved by the large number of 67 reference field sources contributing to the average relative motion between the cluster and the field. The uncertainties were calculated adding the fixed component from

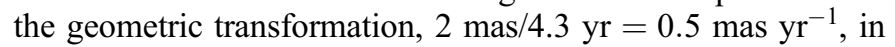
quadrature to the standard deviation in field star velocities, $\sigma_{\text {field }}=$ $1.6 \mathrm{mas} / \mathrm{yr}$, normalized to the number of field stars, $N=67$, $\sigma_{\text {field }} / \sqrt{N}=0.2$ mas yr$^{-1}$. The uncertainty from the zero point defined by the 328 cluster candidates is $0.73 / \sqrt{328}=0.040 \mathrm{mas} / \mathrm{yr}$, and therefore negligible. The final uncertainty from random deviations in the relative motion of $\sigma_{\mu}=\left(0.5^{2}+0.2^{2}\right)^{1 / 2}=$ $0.54 \mathrm{mas} \mathrm{yr}^{-1}$ is dominated by the geometric transformation between NACO and NIRC2, which includes residual optical distortion differences and the resolution-dependent uncertainty from fitting the centroid to the light distribution of each star.

In addition, one might consider that the internal velocity distribution may contribute to the rms scatter in cluster candidate motions. The cluster velocity dispersion can be estimated as $\sigma=$ $\left(0.4 G M_{\mathrm{cl}} / r_{\mathrm{hm}}\right)^{1 / 2}$ (Binney \& Tremaine 1987, eq. [4-80b]), where $M_{\mathrm{cl}}=10^{4} M_{\odot}$ is the total cluster mass and $r_{\mathrm{hm}}=0.4 \mathrm{pc}$ the half-mass radius (Stolte et al. 2002). This yields $\sigma=7 \mathrm{~km} \mathrm{~s}^{-1}$ or $0.2 \mathrm{mas} \mathrm{yr}^{-1}$, which is significantly smaller than the rms scatter

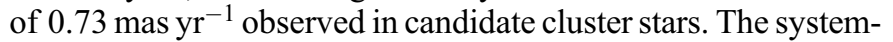
atic uncertainty of $2 \mathrm{mas} / 4.3 \mathrm{yr}$ or $0.5 \mathrm{mas} \mathrm{yr}^{-1}$ imposed from the geometric transformation between NACO and NIRC2 presently limits our ability to detect the internal velocity dispersion of the cluster. With a relative astrometric accuracy of 0.3 mas measured between the three NIRC2 auxiliary frames, the internal velocity dispersion can be observed on a timescale of a few years. A second epoch of NIRC2 data will avoid the transformation uncertainty inherited from the different optical distortions and spatial resolution in the NACO and NIRC2 data sets, thereby decreasing the overall uncertainty in the proper motion measurement, and providing the potential to resolve the internal velocity dispersion of the cluster.

\subsection{Sources of Systematic Uncertainty}

The random uncertainty derived above dominates the proper motion measurement only in the absence of systematic uncertainties. These uncertainties can arise from the choice of selected field stars, as well as a bias toward field sources on the near side of the bulge. In Figure 3 the field selection was based on the density of stars in proper motion space. We can investigate the uncertainty in our measurement due to the field sample selection using defined subsamples. As we expect a larger positional uncertainty for fainter stars, the first sample consists of stars brighter than the apparent cluster main sequence cutoff at $K=17.4$ mag. As bulge stars are predominantly faint, this bright sample consists of 27 sources, and yields $\mu=221 \pm 22 \mathrm{~km} \mathrm{~s}^{-1}$.

As a consequence of the high foreground extinction toward the Galactic center, our observations could be biased to stars on the near side of the bulge. Such a bias should be revealed in a proper motion difference between blue and red sources. Thus we define two sets for our second sample: a set blueward of $H-K=$ $2.1 \mathrm{mag}$, encompassing the cluster main sequence and the bulge red clump, and a very red sample $H-K>2.1$ mag with presumed background stars. The cluster proper motion derived from the "blue" field sample of 45 stars becomes $194 \pm 19 \mathrm{~km} \mathrm{~s}^{-1}$, while the red sample of only 22 stars yields $249 \pm 22 \mathrm{~km} \mathrm{~s}^{-1}$. The larger number of stars in the "blue" sample indicates that there is a bias toward stars on the near side of the bulge in the complete sample. The difference of $55 \pm 29 \mathrm{~km} \mathrm{~s}^{-1}$ is in excellent agreement with the proper motion difference of stars on the near and far side of the bulge, measured using red clump giants to $1.5 \pm 0.1 \mathrm{mas} / \mathrm{yr}$ or $57 \pm 4 \mathrm{~km} \mathrm{~s}^{-1}$ (Sumi et al. 2003). The higher cluster motion of $249 \pm 22 \mathrm{~km} \mathrm{~s}^{-1}$ as measured from the very red 
sources indicates that the bias acts to decrease the cluster velocity. The absolute proper motion might be closer to the mean between the red and blue sample, $\sim 221 \pm 29 \mathrm{~km} \mathrm{~s}^{-1}$, in good agreement with the $212 \pm 20 \mathrm{~km} \mathrm{~s}^{-1}$ derived for the full sample. Nevertheless, the lower number of stars in the red sample indicates that sources with high extinctions or large distances, which have higher relative streaming velocities than sources closer to the $\mathrm{GC}$, are predominantly lost. As this problem arises in all proper motion studies toward the GC, the maximum expected velocity for stars on the far side of the bar is not firmly known. This implies that the proper motion measurement remains a lower limit.

A final concern may arise from the sampling of field stars itself. The bias from the $n=67$ field star sample can be estimated by deriving the cluster motion from a random sample of half of the field stars, $n=34$. In 100 random samples we obtain proper motion values between 195 and $227 \mathrm{~km} \mathrm{~s}^{-1}$, with a mean and standard deviation of $211 \pm 20 \mathrm{~km} \mathrm{~s}^{-1}$, in excellent agreement with the value derived from the full sample above. In summary, we conclude that the only systematic uncertainty in the proper motion might arise from the bias to bulge stars in front of the GC, but the effect is small and the measurements agree very well within the uncertainties. Therefore, we use the full sample of field stars to obtain a conservative lower limit to the proper motion of the cluster in an absolute reference frame, and adjust the uncertainty to account for the possible bias accordingly, $212 \pm 29 \mathrm{~km} \mathrm{~s}^{-1}$.

\subsection{The 3D Motion of the Arches in the Bulge Reference Frame}

In order to derive the absolute motion of the Arches cluster in the Galaxy, we need to understand the field rest frame. The CMD (Fig. 2) shows that most of the proper motion selected field stars are faint and red. The clump of field stars at $K=16, H-K=$ $1.9 \mathrm{mag}$ is consistent with red clump stars at $d=8 \mathrm{kpc}$ and $A_{K}=$ $3 \mathrm{mag}\left(M_{K}=-1.6\right.$; Alves 2000), and the faint population, $K>$ $17 \mathrm{mag}$, covers the regime of evolved bulge stars. Sumi et al. (2003) analyzed a sample of $\sim 50,000$ stars repeatedly observed in OGLE-II fields toward the bulge, and concluded that proper motions of red giants and red clump stars are consistent with zero mean proper motion with respect to the GC. Only blue foreground disk stars display a systematic deviation in mean proper motion from the $\mathrm{GC}$, which is interpreted as the tangential motion of disk stars at 1-2 kpc distance from the Sun. In the NIRC2 field of view, we only observe seven stars significantly bluer ( $H-K<1.3 \mathrm{mag}$ ) than the deeply extincted cluster population ( $A_{V} \sim 24$ mag, Stolte et al. 2002), so that we can safely rule out significant contamination by disk stars.

Sumi et al. (2003) derived the streaming motion of red clump stars in bulge fields from proper motions, and interpret the observed velocity difference of $57 \mathrm{~km} \mathrm{~s}^{-1}$ between sources in front of and behind the GC as the rotation of the galactic bar in the same direction around the GC as the Sun. This streaming motion is consistent with the bar models constructed from CO line-ofsight velocity maps (Binney et al. 1991; Englmaier \& Gerhard 1999). In the context of the $3 \mathrm{D}$ reference frame, a detection bias toward sources on the near side of the bar causes a systematic loss of stars with proper motions in the opposite direction to the Arches motion (compare to the orbit sketches in Fig. 6). The number counts of 22 very red sources as opposed to 45 blue sources indicate a loss of sources at the far side of the bar. The selective detection of sources in the front of the GC shifts the apparent mean motion of the field stars away from zero in the same direction as the Arches cluster, which, when corrected, increases the cluster velocity with respect to the GC even further. The 3D motion derived from the proper motion sample is therefore a lower limit to the space motion of the Arches with respect to the GC. ${ }^{5}$

The heliocentric line-of-sight velocity of the Arches cluster was measured spectroscopically to be $+95 \pm 8 \mathrm{~km} \mathrm{~s}^{-1}$ Figer et al. 2002. Combining the proper motion with the line-of-sight velocity yields the relative three-dimensional space motion of the Arches, $v_{3 \mathrm{D}}=232 \pm 30 \mathrm{~km} \mathrm{~s}^{-1}$, directed away from the GC to the northeast, and away from the Sun.

\section{DISCUSSION AND ORBIT CONSIDERATIONS}

The measurement of the Arches space velocity allows us to compare the cluster velocity with measured orbital velocities of stars and clouds in the inner Galaxy, with the aim of deriving the first qualitative constraints on the cluster orbit around the GC by considering known orbital families in comparison with the 3D cluster motion. The cluster orbit and, in particular, the GC distance are critical for the formation and dynamical evolution of the cluster in the GC potential, for the amount of tidal mass loss of cluster members along its orbit, and for the potential for inspiral toward the GC.

\subsection{Excluding a Circular Orbit?}

Due to the earlier lack of knowledge on the cluster motion and the limited understanding of the inner Galaxy, and in particular the ignoring of the thick nuclear stellar disk between 20 and $200 \mathrm{pc}$ (Launhardt et al. 2002), previous studies of the dynamical evolution and tidal disruption of the Arches in the GC potential assumed for simplicity that the cluster moves on a circular orbit in a spherically symmetric mass distribution (Kim et al. 2000; Portegies Zwart et al. 2002). Without further constraints, a circular orbit in a spherically symmetric potential is the simplest case and most straightforward assumption. From this assumption, the amount of mass loss and the true distance of the Arches from the GC were derived.

In the case of a circular orbit in a spherically symmetric potential, the orbital parameters are determined by the $3 \mathrm{D}$ velocity and projected radius vectors. The circular orbit approximation allows us to compare the derived orbital parameters with observations in the inner Galaxy, and to probe whether a circular orbit assumption is valid for the Arches.

In a spherically symmetric potential, the gravitational force only depends on the mass interior to the present galactocentric radius. While the potential in the inner galaxy is not spherically symmetric, this assumption is made in the concept of the enclosed mass versus radius relation providing the gravitational potential for previous simulations. As shown below, this concept is too simplistic to explain the 3D space motion of the Arches cluster.

For a circular, closed orbit, the absolute distance of the Arches to the $\mathrm{GC}, r_{\mathrm{GC}}$, can be derived geometrically, using $\boldsymbol{v} \cdot \boldsymbol{r}=0$. A convenient coordinate system can be defined from (1) the direction along the proper motion vector $\mu$, (2) the direction perpendicular to $\mu$ on the plane of the sky, and (3) the direction along the line of sight (see Fig. 4). As the 3D velocity of the cluster is defined by $\mu$ and the line-of-sight velocity, $v_{\text {los }}$, the velocity

\footnotetext{
5 Although we cannot fully exclude the possibility that the cluster is observed against a dense comoving group in the background, it appears very unlikely that the majority of field sources would be part of such a group.
} 


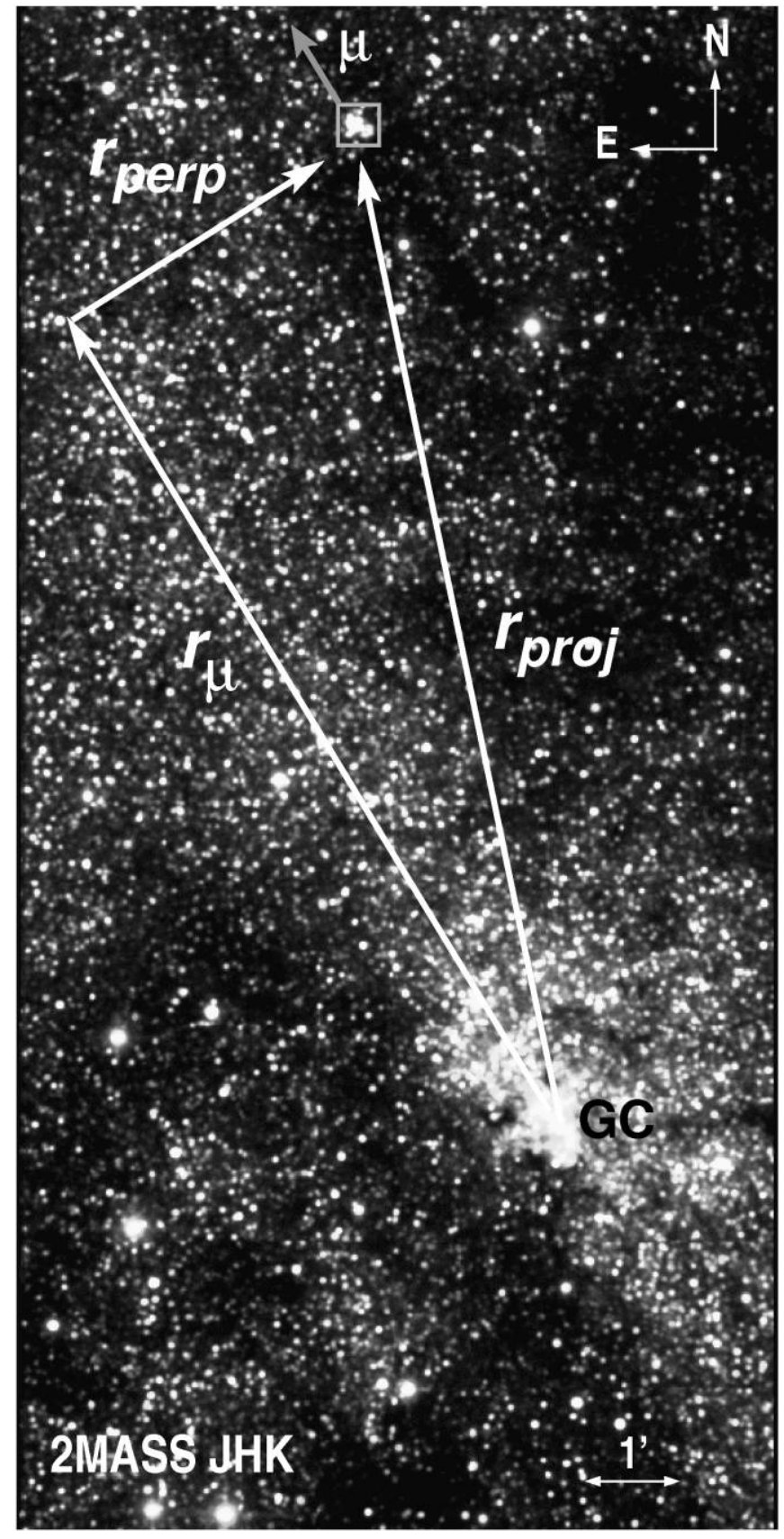

FIG. 4.- Arches cluster proper motion (green arrow) with respect to the GC is displayed on a 2MASS JHK color image. The bright band of stars extending from the GC into the direction of the radius vector $\boldsymbol{r}_{\mu}$ indicates the Galactic plane, with the Arches at a projected height of about $10 \mathrm{pc}$ above the disk. The cluster is moving almost parallel to the plane, and away from the Sun. The vectors illustrate the coordinate system defined to estimate the $3 \mathrm{D}$ distance of the cluster to the GC under the assumption of a circular orbit. [See the electronic edition of the Journal for a color version of this figure.]

component perpendicular to $\mu$ is zero. The circular orbit equation thus becomes

$$
\boldsymbol{v} \cdot \boldsymbol{r}=\mu r_{\mu}+v_{\mathrm{los}} r_{\mathrm{los}}=0
$$

where $r_{\text {los }}$ is the radius vector along the line of sight. With $\mu=$ $212 \pm 29 \mathrm{~km} \mathrm{~s}^{-1}, r_{\mu}=r_{\text {proj }} \cos \phi=25.2 \pm 5.9 \mathrm{pc}$, where $\phi=$ $\arctan \left(v_{\text {los }} / \mu\right)=18.4^{\circ}$ is the angle between the projected radius vector, $r_{\text {proj }}$, and the proper motion vector on the plane of the sky,

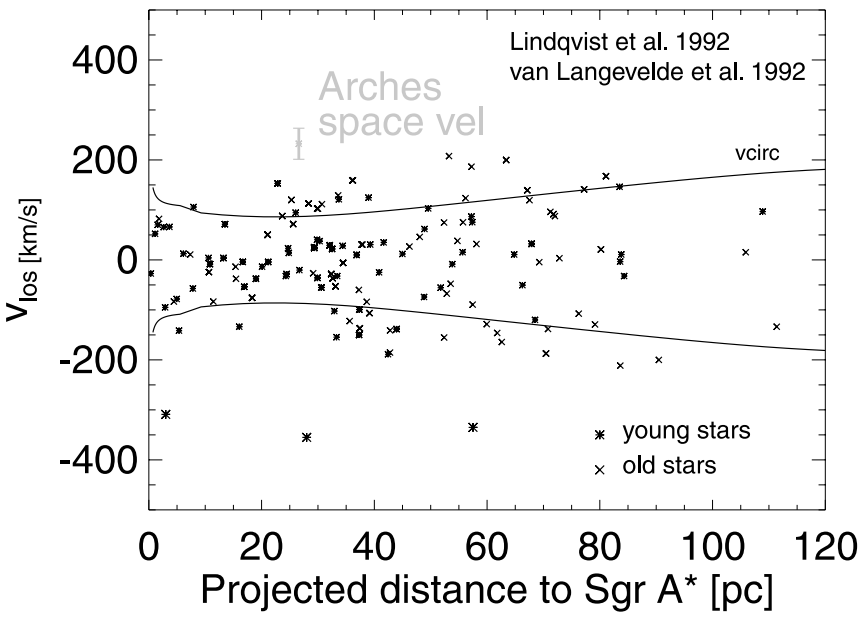

FIG. 5.- Line-of-sight velocity of OH/IR stars observed by Lindqvist et al. (1992). Enveloping lines are orbital velocities expected for circular, Keplerian orbits derived from the enclosed mass (Launhardt et al. 2002). The space motion of the Arches (red asterisk) is significantly larger than expected for a circular, Keplerian orbit at a projected radius of $26 \mathrm{pc}$, implying that the cluster is either at a significantly larger absolute distance to the GC, or is not on a circular orbit, or both. [See the electronic edition of the Journal for a color version of this figure.]

and $v_{\text {los }}=+95 \pm 8 \mathrm{~km} \mathrm{~s}^{-1}$ (Figer et al. 2002), we obtain $r_{\text {los }}=$ $-56.4 \pm 25.5 \mathrm{pc}$, pointing toward us. The GC distance of the cluster in the circular orbit approximation would then be $r_{\mathrm{GC}}=$ $\left(r_{\text {proj }}^{2}+r_{\text {los }}^{2}\right)^{1 / 2}=62 \pm 23$ pc.

As $r_{\mathrm{GC}}$ was derived geometrically, no assumption about the enclosed mass was made. The enclosed mass required to obtain a circular, Keplerian orbit with an orbital velocity of $232 \pm$ $30 \mathrm{~km} \mathrm{~s}^{-1}$ at a radius of $62 \pm 23 \mathrm{pc}$ is $\sim(8 \pm 3) \times 10^{8} M_{\odot}$. However, from the modeled mass distribution in the inner Galaxy, the enclosed mass within $r_{\mathrm{GC}}=62 \mathrm{pc}$ is $2 \times 10^{8} M_{\odot}$ (Launhardt et al. 2002), where the mass contains both interstellar matter and stars as measured from near- to far-IR observations with IRAS and $C O B E$ DIRBE. This discrepancy in the inferred enclosed mass provides a first indication that the Arches moves on a noncircular orbit.

We can further probe the circular orbit approximation by comparing the measured 3D velocity of the cluster with observed and expected velocities in the inner Galaxy. In a sample of circularly orbiting objects along the line of sight, the orbital velocity can be measured as the maximum line-of-sight velocity observed at each projected radius. Following van Langevelde et al. (1992), we reproduce the line-of-sight velocities of a survey of $\mathrm{OH} / \mathrm{IR}$ stars in the inner bulge conducted by Lindqvist et al. (1992) in Figure 5, along with the expected maximum circular velocity at each projected radius derived from the enclosed mass determination by Launhardt et al. (2002). The circular velocities envelop most of the line-of-sight velocity measurements of bulge stars in Figure 5, suggesting that circular motion is a reasonable approximation for stellar orbits in the inner $120 \mathrm{pc}$. Outliers with significantly larger velocities can be understood as stars on highly elongated orbits.

The current orbital velocity of the Arches, $232 \pm 30 \mathrm{~km} \mathrm{~s}^{-1}$, lies significantly above the orbital velocity of $v_{\text {circ }}=86 \mathrm{~km} \mathrm{~s}^{-1}$ expected for a circular orbit at its projected distance of $r_{\mathrm{GC}}=26.6 \mathrm{pc}$ (cf. Fig. 5). The expected $v_{\text {circ }}$ increases to $120 \mathrm{~km} \mathrm{~s}^{-1}$ at $r_{\mathrm{GC}}=$ $62 \mathrm{pc}$, but remains almost a factor of 2 lower than the space velocity of the cluster. The large space velocity of the Arches places the cluster either at very large galactocentric radii, where the Galactic rotation curve flattens $(r \geq 1 \mathrm{kpc})$, which is inconsistent with the cluster's suggested interaction with central molecular zone 

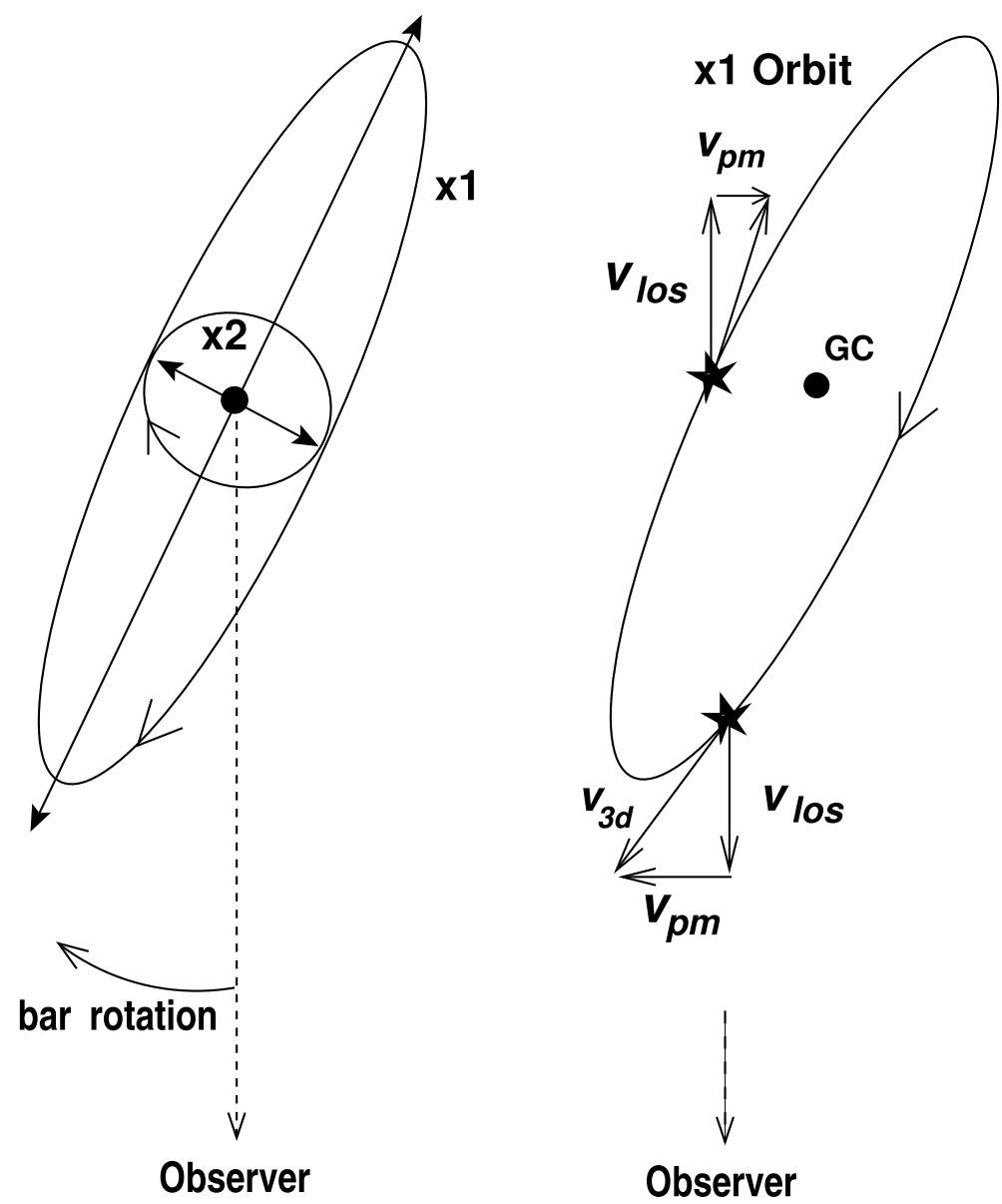

x2 Orbit
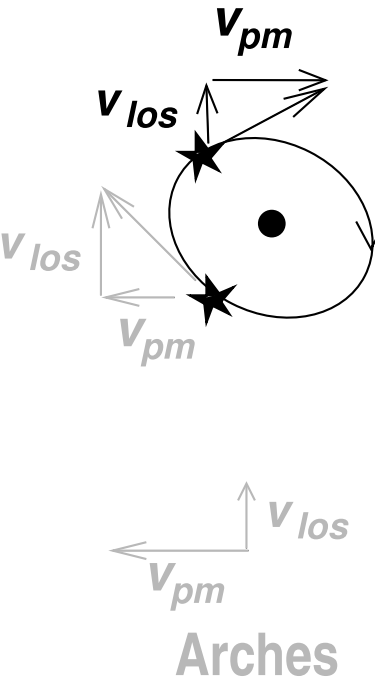

FIG. 6.-Schematic representation of gas cloud orbits in the bar potential in the inner Galaxy. The left panel displays the orientation of $\mathrm{x} 1$ and $\mathrm{x} 2$ orbits with respect to the Galactic bar minor and major axes. The $\mathrm{x} 1$ orbital family reaches out to several $\mathrm{kpc}$, while $\mathrm{x} 2$ orbits are confined to the inner $280 \mathrm{pc}$ of the central bar. The middle panel displays proper motion and line-of-sight velocity vectors expected for objects on $\mathrm{x} 1$ orbits, which are inconsistent with the measured Arches velocity vectors depicted at the lower right (green). The proper motion and line-of-sight velocity directions of objects on x 2 orbits (right panel) are consistent with the observed Arches motion ( green). However, as discussed in $\S 4.2$, the magnitude of the cluster velocity is too large for expected $\mathrm{x} 2$ orbital velocities. [See the electronic edition of the Journal for a color version of this figure.]

(CMZ) clouds ( Lang et al. 2003; Wang et al. 2006), or suggests a noncircular orbit. A noncircular orbit would also explain the overestimated enclosed mass derived above. ${ }^{6}$

A circular cluster orbit in a spherically symmetric potential is inconsistent with the measured enclosed mass at the radius $r_{\mathrm{GC}}$ and with observed circular velocities in the distance range considered for the Arches.

\subsection{Relationship to Gas Cloud Orbits: A Scenario for the Origin of the Arches}

In the central region of the Galaxy, the two-body relaxation time for a cluster with $M_{\mathrm{cl}}=10^{4} M_{\odot}$ embedded in a medium of stellar density $\rho \sim 4 \times 10^{2} M_{\odot} \mathrm{pc}^{-3}$ (Launhardt et al. 2002) is on the order of a few $10^{9} \mathrm{yr}$ (following Binney \& Tremaine 1987, eq. [8-71]). With an age of only $2.5 \mathrm{Myr}$, the cluster velocity should therefore carry the imprint of the motion of its native cloud. A comparison of the cluster space motion with the characteristic motion of gas clouds in the inner Galaxy might shed light on its

\footnotetext{
${ }^{6}$ Three OH/IR stars with similarly high velocities of $\sim 200 \mathrm{~km} \mathrm{~s}^{-1}$ are observed between projected radii of 53 to $65 \mathrm{pc}$. These stars could have been ejected from close binary systems or dense clusters such as the Quintuplet or the Arches itself. While stars in the high source density of the inner Galaxy have short twobody relaxation timescales, a massive cluster is not easily deflected from a regular orbit.
}

origin. In the central $l= \pm 2^{\circ}$ of the Galaxy, the outer envelope of the $\mathrm{CO}$ emission in the longitude, line-of-sight velocity $\left(l-v_{\text {los }}\right)$ plane displays a "parallelogram" structure, which is interpreted as the reaction of the gas to a bar potential (Binney et al. 1991; Englmaier \& Gerhard 1999). Depending on their distance from the GC, long-lived gas clouds are suspected from these models to move on noncircular, closed orbits in the corotating reference frame of the bar, which belong to the $\mathrm{x} 1$ orbit family aligned with the major axis of the bar and the $\mathrm{x} 2$ orbit family aligned with the bar's minor axis (see Fig. 6). Cloud motion at large GC radii is dominated by $\mathrm{x} 1$ orbits with line-of-sight velocities up to $270 \mathrm{~km} \mathrm{~s}^{-1}$ outside $l>2^{\circ}\left(r_{\mathrm{GC}}=280 \mathrm{pc}\right)$, while inside the corresponding radius $\mathrm{x} 2$ orbits are expected to populate the inner spheroid.

Dame et al. (2001) observe line-of-sight velocities of CO gas up to $230 \mathrm{~km} \mathrm{~s}^{-1}$ close to $l=2^{\circ}$. While the Arches velocity would be consistent with the peak velocities observed outside $l=2^{\circ}$, for objects on $\mathrm{x} 1$ orbits at positive longitudes and with positive proper motion (toward increasing $l$ ), negative line-of-sight velocities should be observed, which is not the case (Fig. 6). The positive $v_{\text {los }}$ observed for the Arches agrees in sign with $\mathrm{x} 2$ orbit simulations, but the high space velocity is inconsistent with the maximum $\mathrm{x} 2$ orbital velocity of $120 \mathrm{~km} \mathrm{~s}^{-1}$ suggested by the Englmaier \& Gerhard bar model. Thus, it appears that the cluster is not on one of these orbital families suggested for gas clouds in the inner Galaxy. 
How can the cluster have gained such a high space velocity at such small GC distance? Binney et al. (1991) point out that gas clouds with substantial molecular components are only observed on the innermost $\mathrm{x} 1$ orbit $\left(r_{\mathrm{GC}} \sim 280 \mathrm{pc}\right)$ and the $\mathrm{x} 2$ orbits at even smaller radii, but not in the outer bar. They provide the intriguing interpretation that gas clouds on inner $\mathrm{x} 1$ orbits may collide with clouds on the outermost $\mathrm{x} 2$ orbits, where the cloud density is higher. The subsequent fate of the clouds depends on the impact angle. Close to the $\mathrm{x} 2$ apocenter, clouds might merge and lose kinetic energy in the process. In a less direct collision, SPH simulations by Athanassoula (1989; reproduced in Binney et al. 1991, their Fig. 6) suggest that the $x 1$ cloud will be dispersed into the $\mathrm{x} 1-\mathrm{x} 2$ transition zone, and collide with incoming material on the opposite side. In both cases, cloud material will experience shock compression. Binney et al. suggest that the collision causes shockcreation of molecules, and the friction might allow the remnant $\mathrm{x} 1$ cloud to shed angular momentum and spiral in toward a stable $\mathrm{x} 2$ orbit.

If this scenario holds for sufficiently massive clouds, we can speculate that the $\mathrm{x} 1-\mathrm{x} 2$ collision could trigger a starburst event, which would take place during the inspiral of the shocked cloud toward the region of stable $\mathrm{x} 2$ orbits. The emerging stellar cluster would naturally inherit the cloud velocity depending on the point along the $\mathrm{x} 1-\mathrm{x} 2$ orbit transition where the massive stars evaporate the native material. Once the cluster emerged from its native cloud, it is unaffected by collisions with ambient clouds, and can lose energy solely by the slow process of two-body relaxation. At this point, the cluster can sustain a significant velocity component from the higher $\mathrm{x} 1$ orbital velocity. This scenario could explain both the apparent orientation of the Arches velocity vectors in agreement with $\mathrm{x} 2$ orbits, and the high velocity preserved from the $\mathrm{x} 1$ orbit transition.

Although the dynamical properties of the cluster are well explained, this theory has a significant caveat. For the Arches to inherit the high $\mathrm{x} 1$ velocity, the $\mathrm{x} 1$ cloud would have to be more massive and dense than the $\mathrm{x} 2$ cloud, which contradicts the distribution of dense clouds observed in the CMZ today. The presentday clouds on $\mathrm{x} 1 / \mathrm{x} 2$ orbits representing the bar potential are measured in $\mathrm{CO}$, which traces gas at average densities $n_{\mathrm{H}_{2}} \sim$ $10^{3} \mathrm{~cm}^{-3}$, suspected to be too low for stellar fragmentation. The denser components with $n_{\mathrm{H}_{2}} \geq 10^{4}-10^{5} \mathrm{~cm}^{-3}$ capable of intense star formation are efficiently detected in the CS $J=(1-0)$ transition. While CS is correlated with many of the intense $\mathrm{CO}$ peaks (Bally et al. 1988), CS clouds are more confined to the inner parts of the $\mathrm{CMZ}\left(r_{\mathrm{GC}}<200 \mathrm{pc}\right)$ and display maximum line-of-sight velocities $v_{\text {los }}<120 \mathrm{~km} \mathrm{~s}^{-1}$. These velocities, if representative of the orbital velocities of dense clouds, are consistent with clouds on $\mathrm{x} 2$ orbits, but appear inconsistent with the large orbital velocity measured for the Arches. While the large present-day velocity of the Arches is suggestive of the collision scenario, we need to understand the initial conditions at the time of the starburst to constrain whether dense clouds could have been triggered to form the cluster, and in particular whether a large orbital velocity at the present projected position can be acquired from these initial conditions. For this purpose, we perform a backward integration of the cluster motion in the observed, non-spherically symmetric extended mass distribution in the inner Galaxy.

\section{THE ARCHES MOTION IN THE GC POTENTIAL}

The knowledge of the three-dimensional space motion of the cluster and the projected distance from the GC allow us to investigate possible orbits of the cluster in the potential of the inner Galaxy depending on the cluster's line-of-sight distance. The cir- cular orbit considerations in $\S 4$ suggest that motion on a circular orbit in a spherically symmetric potential cannot explain the high $3 \mathrm{D}$ velocity of the cluster. As the stellar density in the inner Galaxy changes dramatically with radius, we exploit the knowledge of the existing density of matter to approximate a more realistic gravitational potential. The cluster is evolved in this potential to yield constraints on its present location with respect to the GC, the possible position on its orbit, and its origin. ${ }^{7}$

\subsection{Modeling the Potential in the Inner Galaxy}

Three components are suspected to contribute to the mass distribution inside $200 \mathrm{pc}$, namely, the central black hole, the nuclear stellar cluster, and the thick nuclear disk comprised of both gas and stars. From their observed mid- to far-infrared luminosity distributions, Launhardt et al. (2002) describe the density profiles of the nuclear stellar cluster as a spherically symmetric power-law decline, and the nuclear disk as a combination of two exponentials with additional flattening in the latitude direction. Beyond $200 \mathrm{pc}$, the observed nuclear disk declines rapidly and the bar becomes dominant according to the observations. In the central few parsecs, the black hole is also an important contributor to the potential, and is added as a point mass with $M=3.6 \times 10^{6} M_{\odot}$.

As the only free variable of the cluster velocity and space coordinates is the distance along the line of sight, we define a sky coordinate system from the observer's perspective with $x$ the projected distance from the GC along the Galactic plane, $y$ the line-of-sight distance, and $z$ the height above the Galactic plane. The line-of-sight distance is defined such that zero corresponds to the distance of the GC from the Sun. A positive line-of-sight distance implies the present cluster location is behind the GC. Because the proper motion vector is oriented parallel to the Galactic plane, this coordinate system is similar to the geometric system defined in $\S 4$ to estimate the circular orbit, where the $x$-axis is parallel to the proper motion vector, and the $y$-coordinate represents the unconstrained radius vector along the line of sight.

As the bar is inclined by $25^{\circ}$ toward positive longitude with respect to the line of sight (Rattenbury et al. 2007), ${ }^{8}$ while all other components are axisymmetric, we convert the initial cluster position and velocity into the bar system, with $x_{b}$ along the major axis and $y_{b}$ along the minor axis of the bar, while the height above the plane, $z$, is not modified. After evolving the cluster motion in the combined potential, the output positions, velocities, and accelerations are converted back to the sky coordinate system.

In order to calculate the combined gravitational potential at every position, we use logarithmic potentials with different stretching parameters to represent these components. The basic form of the adopted logarithmic potential is

$$
\Phi=0.5 v_{0}^{2} \log \left(R_{c}^{2}+\frac{x_{b}^{2}}{a^{2}}+\frac{y_{b}^{2}}{b^{2}}+\frac{z^{2}}{c^{2}}\right),
$$

\footnotetext{
7 We use a leap-frog integrator originally developed by Joshua Barnes for a single logarithmic potential, which was extensively adapted to represent the gravitational components in the inner Galaxy. The basic version of the orbit integrator is publicly available from his lecture notes (see http://www.ifa.hawaii.edu/ barnes for more information).

${ }^{8}$ The bar rotation angle changes with the pattern speed of $60 \pm 5 \mathrm{sr} \mathrm{Gyr}^{-1}$ (Bissantz et al. 2003), implying a rotation of $9^{\circ}$ in 2.5 Myr. On prograde orbits, the travel time of the cluster through the corotating bar potential becomes longer, while the travel time through the bar is shortened in retrograde orbits, causing orbits at large $\mathrm{GC}$ distances $\left(r_{\mathrm{GC}}>200 \mathrm{pc}\right)$ to rotate in the same direction as the bar with respect to the line of sight. As the effect during the cluster lifetime is small and the most likely location of the cluster is inside $200 \mathrm{pc}$, we do not account for bar rotation in this model.
} 


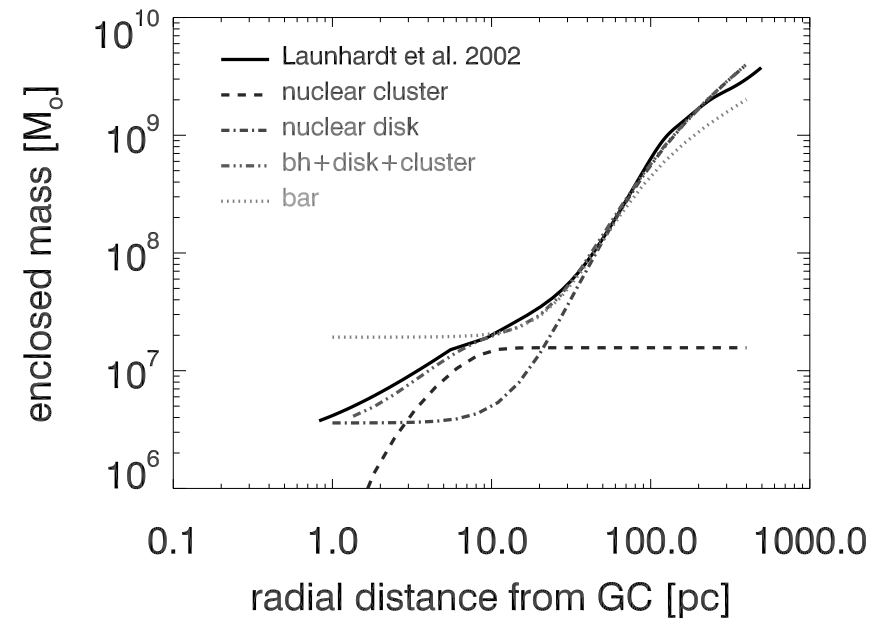

FIG. 7.-Fit to the observed enclosed mass (Launhardt et al. 2002; see also Lindqvist et al. 1992). In the central $200 \mathrm{pc}$, the gravitational potential is represented by a spherical component, the nuclear stellar cluster, and an axisymmetric component flattened in galactic latitude, the nuclear stellar disk. The black hole is added as a point mass. The estimated enclosed mass from the combined potentials $(\mathrm{BH}+$ disk + cluster) reproduces the enclosed mass very well. Beyond $200 \mathrm{pc}$, the potential is represented by an elongated bar. The bar slightly underestimates the enclosed mass, because during the transition from the inner to the outer potential a continuous acceleration is enforced to avoid nonphysical behavior. [See the electronic edition of the Journal for a color version of this figure.]

where $v_{0}^{2}$ represents the velocity on the flattened portion of the rotation curve at very large radii, and $R_{c}$ the core radius. Both constants are free parameters in the fit to the observed mass distribution. The stretching parameters $a, b$, and $c$ describe the flattening of the potential along the $x_{b}, y_{b}$, and $z$-axes, respectively, with respect to a spherically symmetric potential where $a=b=$ $c=1.0$. For the axisymmetric components representing the central cluster and thick disk this definition implies $a=b=1.0$. In order to derive $v_{0}^{2}$ and $R_{c}$ for each component, we spatially integrate the modeled density distribution corresponding to the logarithmic nuclear disk and cluster potentials to fit the observed enclosed mass in the central 200 pc (Launhardt et al. 2002, their Fig. 14). Additional constraints are given by the requirement that the acceleration, i.e., the first spatial derivatives of the potential, be continuous. The best mass fit under these conditions is shown in Figure 7, and the adopted parameters for each component are summarized in Table 1.

In our adopted model, the nuclear cluster is the dominant mass component between 2 and $7 \mathrm{pc}$, beyond which it is smoothly truncated to a constant mass, and the thick nuclear disk becomes the dominant component beyond $20 \mathrm{pc}$, while both contribute significantly to the mass at intermediate radii. The sum of all three components, including the black hole, provides a very good approximation to the best available estimate of the enclosed mass in the inner 200 pc of the Galaxy.

The transition between the inner components and the bar potential poses the largest problem for computational continuity. In order to ensure that the acceleration vary smoothly and the energy be conserved along the orbit, the disk is transformed into an elongated bar potential in the transition zone between 150 and $250 \mathrm{pc}$. Technically, a smooth variation of the potential is achieved by multiplying a sigmoid function of the form $1 /\left(1+e^{-r / f}\right)$ to the stretching parameters. Here, $r=r_{\mathrm{GC}}-r_{\text {trans }}$ is the difference between the radial distance from the GC and the midpoint of the transition region, $r_{\text {trans }}=200 \mathrm{pc}$, and $f$ is a scaling factor that defines the steepness of the transition. The sigmoid function is 0 for radii significantly below the transition radius $r_{\text {trans }}$, and 1 for radii
TABLE 1

Parameters for Nuclear Cluster, Disk, and Bar in the Logarithmic Potential Approximation

\begin{tabular}{|c|c|c|c|c|c|}
\hline Potential & $a$ & $b$ & $c$ & $\begin{array}{c}R_{c} \\
(\mathrm{pc})\end{array}$ & $\begin{array}{c}v_{0} \\
\left(\mathrm{~km} \mathrm{~s}^{-1}\right)\end{array}$ \\
\hline Nuclear stellar cluster ................ & 1.0 & 1.0 & 1.0 & 2.0 & 98.6 \\
\hline Nuclear stellar disk .................... & 1.0 & 1.0 & 0.71 & 90 & 190 \\
\hline Bar & 0.75 & 1.0 & 0.75 & 90 & 190 \\
\hline
\end{tabular}

significantly above this radius. The scaling factor is chosen to be $f=15$, which ensures a gentle transition over the full range of radii, $150<r_{\text {trans }}<250 \mathrm{pc}$. For a smooth transition between the nuclear disk and the bar potential, we therefore modify the $x$-stretching and $z$-stretching parameters from the axisymmetric disk with $a_{\text {disk }}=1.0$ and $c_{\text {disk }}=0.71$ to become the elongation of the bar, $a_{\mathrm{bar}}=c_{\mathrm{bar}}=0.75$ (Binney et al. 1991), ${ }^{9}$ as follows:

$$
\begin{aligned}
& a(r)=a_{\mathrm{disk}}-\left(a_{\mathrm{disk}}-a_{\mathrm{bar}}\right) /[1+\exp (-r / 15)], \\
& c(r)=c_{\mathrm{disk}}+\left(c_{\mathrm{bar}}-c_{\mathrm{disk}}\right) /[1+\exp (-r / 15)],
\end{aligned}
$$

while $b$ remains 1.0 in the bar reference frame. At any given position, the local conversion factor between the disk and the bar is taken into account in the calculation of the potential and the acceleration, and the subsequently derived velocity and position vector. Beyond ensuring mathematical continuity, the smooth transformation of the thick disk into the bar can be interpreted as one continuous potential in the inner Galaxy, which is known to be disklike in the central region and barlike at larger radii, while the exact shape of the potential combining the two components is unknown.

\subsection{Constraints on the Cluster Orbit}

The family of orbits of the Arches cluster was calculated over a range of line-of-sight distances to the GC from -500 to $500 \mathrm{pc}$. The absolute distance is not well constrained, even if formation scenarios for GC clusters suggest the cluster to be within the inner $200 \mathrm{pc}$. The most stringent evidence for a location close to the GC of the Arches are (1) the mean foreground extinction of $A_{V}=27 \mathrm{mag}$, which is consistent with the line-of-sight extinction toward the GC (Nagata et al. 1995; Cotera et al. 1996), and (2) the suggested interaction of the cluster with clouds in the central molecular zone in the inner $\sim 200$ pc of the Galaxy (Wang et al. 2006; Lang et al. 2003). We note that (1) provides only a weak constraint on the Arches distance due to the strong spatial variability of the extinction observed in the GC environment. For example, in the central parsec of the nuclear stellar cluster, extinction values between $24<A_{V}<34$ mag are measured (Schoedel et al. 2007). We thus decided to cover the largest plausible distance range taking into account these constraints.

Orbits were calculated in steps of $10 \mathrm{pc}$ along the line of sight both forward and backward in time. Backward orbits are considered over the 2.5 Myr since the formation of the cluster to

\footnotetext{
9 Binney et al. (1991) derived an elongation of 0.75 by modeling line-of-sight velocities of gas clouds in the inner Galaxy. More recent determinations of the bar potential from red clump stars suggest a significantly stronger bar elongation with a stretching factor of $\sim 0.3$ (Bissantz \& Gerhard 2002; Rattenbury et al. 2007). The minimum stretching parameter allowed in the analytic density solution of a logarithmic potential is 0.71 to ensure a positive density at all positions. A more realistic, elongated bar potential requires the full numerical integration of the density distribution to obtain the cluster orbit, which is beyond the scope of this paper. We therefore adopt the $q$ parameter derived from cloud velocities. This choice only affects cluster orbits beyond $r_{\mathrm{GC}}= \pm 200 \mathrm{pc}$.
} 

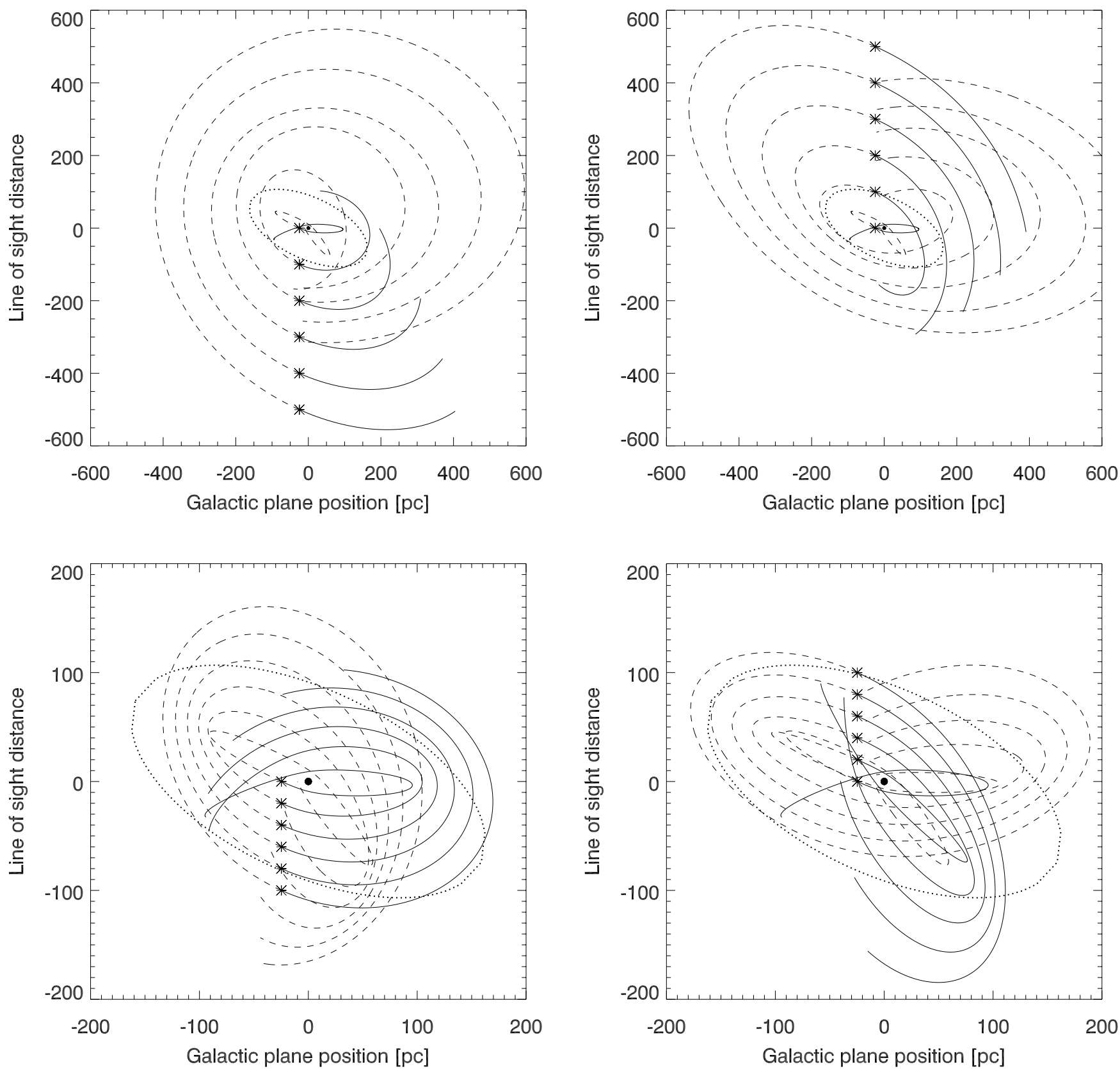

FIG. 8.- Selected orbits in the combined central potential. The orbits are viewed from above the Galactic plane, with the $x$-axis displaying the projected distance from the Galactic center in the direction of the Galactic plane, and the $y$-axis displaying the line-of-sight distance to the observer. The asterisks mark the present location of the cluster for various line-of-sight distances. The GC is located at the origin. The solid lines represent the backward integration of the Arches velocity to the possible cluster origin 2.5 Myr ago. The dashed lines show the extrapolated future orbit to one full $360^{\circ}$ revolution around the GC. The nuclear stellar cluster and disk, including the black hole, are relevant out to $200 \mathrm{pc}$, beyond which the bar potential dominates. The location of the outermost $\mathrm{x} 2$ orbit for a bar inclination of $25^{\circ}$ is indicated by the dotted line (model from Bissantz et al. 2003).

constrain possible initial conditions. As most of the allowed cluster orbits in the asymmetric potential are not closed, we define one orbit as a change in position angle of $360^{\circ}$. In the following discussion, we will refer to parameters originating $2.5 \mathrm{Myr}$ ago as initial conditions assumed to be close to the time of cluster formation, and to parameters projected from the cluster's present position along the first full orbit into the future as the next orbit. The results are presented in Figures 8-11 and Tables 2 and 3.

The uncertainty in the orbital parameters was estimated with two modified orbital simulations, in addition to the standard model represented in Table 1. In order to probe the effects of the shape of the potential, one set of orbits was simulated in a more spherical potential, with $a=c=0.8$ for the bar and $c=0.8$ for the disk. Note that our standard model $c=0.71$ is the flattest logarithmic potential available without obtaining negative densities in the central region. In the modified potential, the apocenter and pericenter distances, minimum and maximum velocities along the initial and next orbit, as well as the period, were only marginally different from the standard model. We thus do not show the results here, as they are indistinguishable from the parameters shown in Figures 8-11. In a second test, the cluster proper motion, the largest velocity component contributing most of the uncertainty in the cluster motion, was modified to $\mu \pm \sigma_{\mu}=212 \pm$ $29 \mathrm{~km} \mathrm{~s}^{-1}$, from 183 to $241 \mathrm{~km} \mathrm{~s}^{-1}$. The largest deviation in both apocenter/pericenter distances and orbital velocities occurs if the cluster's present line-of-sight distance is larger than $200 \mathrm{pc}$. 

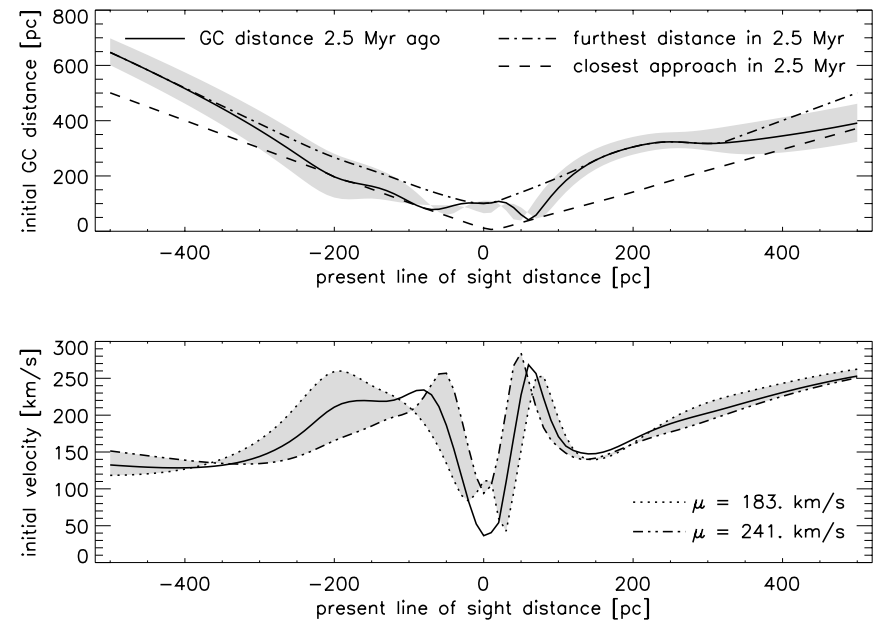

FIG. 9.- Initial orbital parameters of the Arches cluster at the suspected time of birth $\sim 2.5$ Myr ago. The top panel displays the initial GC distance, which would have been the distance of the cluster-forming cloud at the time when the starburst occurred, while the bottom panel shows the initial cluster velocity. Both the furthest and closest approach of the cluster to the GC depend almost linearly on its present-day GC distance. The asymmetries are due to the inclined bar potential. The uncertainty of $\pm 29 \mathrm{~km} \mathrm{~s}^{-1}$ in the proper motion as propagating into the orbital parameters are indicated by the underlying gray area, which was derived from limiting models with $\mu_{\min }=183 \mathrm{~km} \mathrm{~s}^{-1}$ and $\mu_{\max }=241 \mathrm{~km} \mathrm{~s}^{-1}$.

In general, the orbital characteristics are very similar when a larger or smaller present-day proper motion is assumed. These test cases confirm that the derived orbits are not very sensitive to details in the logarithmic potential or the uncertainties in the cluster velocity.

Sample orbits of the cluster in the Galactic plane are shown in Figure 8. Only the motion in the plane is shown, which is superposed on a small oscillation of less than $50 \mathrm{pc}$ above and below the plane. For clarity, orbits originating from a cluster position in front of the GC (left panels) are shown separately from present-day positions behind the GC (right panels). It is noteworthy that, due to the positive velocity along the line of sight, all orbits where the cluster is presently behind the $\mathrm{GC}$ are retrograde to the general motion of stars and gas clouds in the bar potential, and all orbits where the cluster is presently in front of the GC are prograde. If
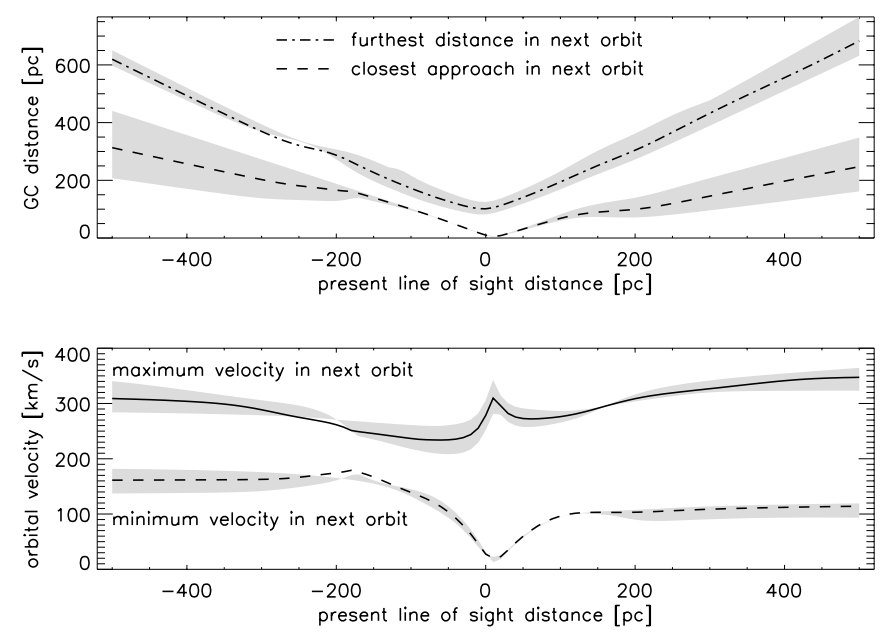

FIG. 10.-Extrapolated next orbit of the Arches cluster around the GC. The top panel shows the closest and furthest distance from the GC during the next orbit. The bottom panel displays the minimum and maximum orbital velocities the cluster can attain on its orbital path. The gray area indicates uncertainties in the orbital simulations from the uncertainty in the proper motion of $\pm 29 \mathrm{~km} \mathrm{~s}^{-1}$.

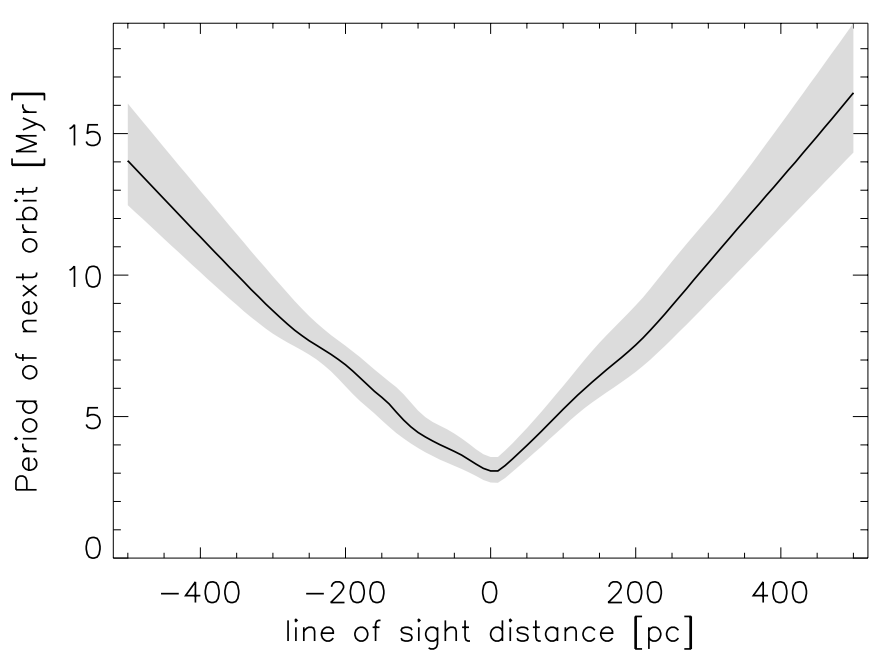

FIG. 11.- Period vs. line-of-sight distance. The extrapolated period of the next full orbit of the Arches cluster in the asymmetric GC potential. As in Figs. 9 and 10 , the gray area indicates the uncertainty in the orbital parameters from the $1 \sigma$ uncertainty in the cluster velocity.

the cluster formed from a cloud on a prograde orbit, it has to be located in front of the GC today. For all present-day locations inside $r_{\mathrm{GC}}<100 \mathrm{pc}$, the high velocity suggests that the cluster recently passed the pericenter of its orbit and now moves toward apocenter.

The approximate shape and location of the outermost $\mathrm{x} 2$ orbit as suggested by the recent bar model of Bissantz et al. (2003) is shown as a dotted ellipse in Figure 6 (top panels). For a cluster location in front of the GC today, a line-of-sight distance of $100-200 \mathrm{pc}$ is most consistent with a formation locus near the outermost $\mathrm{x} 2$ orbit as suggested by $\mathrm{x} 1-\mathrm{x} 2$ cloud collision scenarios. Such a formation scenario is less likely for orbits where the cluster is found behind the GC at present. At line-of-sight distances larger than $200 \mathrm{pc}$, the cluster origin seems unrelated to the $\mathrm{x} 1-\mathrm{x} 2$ transition zone. Triggered cluster formation from dispersed $\mathrm{x} 1$ clouds entering the $\mathrm{x} 2$ orbital zone is also feasible if the cluster is inside the inner $100 \mathrm{pc}$ of the Galaxy, both for line-of-sight distances in front of and behind the GC. Although the direct comparison of the cluster formation locus and the outermost $\mathrm{x} 2$ orbit is complicated by the uncertainty of $\pm 0.5 \mathrm{Myr}$ in the cluster age and thus its exact origin, as well

TABLE 2

Orbital Parameters in the Past 2.5 Myr vs. Present-Day Line-of-Sight Distance

\begin{tabular}{|c|c|c|c|c|}
\hline \multirow{2}{*}{$\begin{array}{l}r_{\mathrm{los}} \\
(\mathrm{pc})\end{array}$} & \multirow{2}{*}{$\begin{array}{l}\text { INITIAL } v \\
\left(\mathrm{~km} \mathrm{~s}^{-1}\right)\end{array}$} & \multicolumn{3}{|c|}{$r_{\mathrm{GC}}(\mathrm{pc})$} \\
\hline & & Initial & Minimum & Maximum \\
\hline-500 & 132 & 647 & 501 & 647 \\
\hline-400 ....................... & 128 & 516 & 401 & 518 \\
\hline ………......... & 144 & 367 & 301 & 389 \\
\hline …………...... & 211 & 197 & 197 & 267 \\
\hline-100 & 229 & 107 & 103 & 172 \\
\hline-50 & 186 & 86 & 56 & 127 \\
\hline 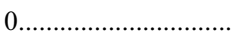 & 36 & 100 & 11 & 101 \\
\hline $50 \ldots \ldots \ldots \ldots \ldots \ldots \ldots \ldots \ldots \ldots \ldots$ & 227 & 60 & 31 & 141 \\
\hline 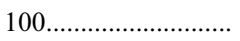 & 171 & 156 & 69 & 194 \\
\hline $200 \ldots \ldots \ldots \ldots \ldots \ldots \ldots \ldots$ & 167 & 305 & 142 & 305 \\
\hline 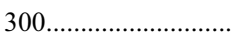 & 203 & 317 & 221 & 317 \\
\hline $400 \ldots \ldots \ldots \ldots \ldots \ldots \ldots$ & 231 & 345 & 296 & 401 \\
\hline $500 \ldots \ldots \ldots \ldots \ldots \ldots \ldots \ldots \ldots \ldots \ldots \ldots$ & 253 & 391 & 372 & 501 \\
\hline
\end{tabular}


TABLE 3

Orbital Paramters of the Next Full Orbit vs. Present Line-of-Sight Distance and Absolute GC Distance, $r_{\mathrm{GC}}$, for Each Orbit

\begin{tabular}{|c|c|c|c|c|c|c|}
\hline \multirow{2}{*}{$\begin{array}{l}r_{\mathrm{los}} \\
(\mathrm{pc})\end{array}$} & \multirow{2}{*}{$\begin{array}{l}r_{\mathrm{GC}} \\
(\mathrm{pc})\end{array}$} & \multicolumn{2}{|c|}{$r_{\mathrm{GC}}(\mathrm{pc})$} & \multicolumn{2}{|c|}{$v\left(\mathrm{~km} \mathrm{~s}^{-1}\right)$} & \multirow{2}{*}{$\begin{array}{l}\text { PERIOD } \\
\text { (Myr) }\end{array}$} \\
\hline & & Minimum & Maximum & Minimum & Maximum & \\
\hline$-500 \ldots \ldots$. & 501 & 313 & 620 & 161 & 310 & 14.0 \\
\hline$-400 \ldots \ldots$. & 401 & 257 & 493 & 162 & 304 & 11.4 \\
\hline$-300 \ldots \ldots$. & 301 & 203 & 369 & 163 & 289 & 8.7 \\
\hline$-200 \ldots \ldots$. & 202 & 166 & 287 & 174 & 262 & 6.8 \\
\hline$-100 \ldots \ldots$. & 103 & 100 & 172 & 139 & 237 & 4.4 \\
\hline$-50 \ldots \ldots \ldots$ & 57 & 56 & 127 & 104 & 234 & 3.8 \\
\hline $0 \ldots \ldots \ldots \ldots . . . . .$. & 27 & 10.0 & 101 & 28 & 278 & 3.1 \\
\hline $50 \ldots \ldots \ldots \ldots$ & 57 & 31 & 141 & 60 & 272 & 4.0 \\
\hline $100 \ldots \ldots \ldots$ & 103 & 68 & 194 & 96 & 276 & 5.3 \\
\hline $200 \ldots \ldots \ldots . . .$. & 202 & 99 & 304 & 103 & 309 & 7.5 \\
\hline $300 \ldots \ldots \ldots . . .$. & 301 & 145 & 432 & 109 & 327 & 10.4 \\
\hline $400 \ldots \ldots \ldots . . .$. & 401 & 197 & 556 & 112 & 341 & 13.4 \\
\hline $500 \ldots \ldots \ldots$ & 501 & 247 & 683 & 114 & 347 & 16.4 \\
\hline
\end{tabular}

as by the extent of the outermost $\mathrm{x} 2$ orbit populated by dense clouds and the uncertainty in the bar orientation angle, the suggested scenarios support the origin of the Arches inside the central 200 pc.

The initial conditions of the molecular cloud at the time when the starburst occurred $\sim 2.5 \mathrm{Myr}$ ago are depicted in Figure 9. The general trend suggests that the distance of closest and furthest approach to the GC in the past $2.5 \mathrm{Myr}$ both scale linearly with the present-day distance of the cluster from the GC. The asymmetries originate from the passage through the inclined bar potential. When the cluster is presently located in front of the GC, it approaches the bar and its path was not strongly influenced by the bar potential in the past 2.5 Myr. If, on the other hand, the cluster is located behind the $\mathrm{GC}$, it had to pass through the bar recently and experienced the increased gravitational forces from the central bar potential. In the latter case, the Arches is now moving away from the major axis of the bar and will not encounter the region of highest density in the bar in the near future. The absolute minimum of the distance to the GC occurs when the Arches is on a radial orbit. Given the orientation of the measured velocity vector, the Arches would have to be at a line-of-sight distance of about $20 \mathrm{pc}$ behind the GC today to be on a radial orbit. On nearly radial orbits, the Arches would have come closer than $10 \mathrm{pc}$ to the GC in the past, thereby likely suffering intense tidal stripping. Such tidal stripping should be detectable in a strong tail of tidal debris distributed along the cluster orbit. Once stars are not bound to the cluster potential anymore, the relevant two-body relaxation time shrinks to the stellar two-body relaxation time of $\sim 10^{7} \mathrm{yr}$ in the nuclear cluster. One might therefore expect that tidally stripped stars suffer dynamical interactions with nuclear cluster sources, which may lead to a rapid dispersal of the tidal tail during future passages through the nuclear cluster. Kim \& Morris (2003) simulate the dispersal of a cluster with an initial core density of $10^{5}-10^{6} M_{\odot} \mathrm{pc}^{-3}$ starting at $30 \mathrm{pc}$ on a highly elongated orbit. Such a cluster can deposit at most $5 \%-10 \%$ of its stellar population at radii $r<5 \mathrm{pc}$ inside the nuclear cluster, and practically no stars in the central parsec. Thus, even if the Arches is on a radial orbit, we do not expect to find Arches stars near the supermassive black hole (SMBH).

Of all orbits, the only ones which can bring the cluster into the inner $10 \mathrm{pc}$ of the GC in the past $2.5 \mathrm{Myr}$, require a present-day GC distance of $r_{\mathrm{GC}}<33 \mathrm{pc}$, very close to its observed projected distance. Any larger distance suggests that the estimated total stel- lar mass of $\sim 10^{4} M_{\odot}$ (Stolte et al. 2002) is very close to the initial cluster mass. As inspiraling cluster models require initial masses of $M_{\mathrm{cl}} \geq 10^{6} M_{\odot}$ from a distance of $30 \mathrm{pc}$ (Kim \& Morris 2003), the orbital analysis observationally confirms the numerical prediction (Kim et al. 2000) that the initial mass of the Arches was never substantial enough for inspiral into the central few parsecs.

Characteristics of the projected future orbit of the Arches are shown in Figure 10. The top panel shows the distance of closest and furthest approach, suggesting that, regardless of its origin, the cluster can approach the GC to within $200 \mathrm{pc}$ during the next few Myr. The approximately linear dependence of closest approach on present line-of-sight distance implies that without energy loss, the cluster cannot get much closer to the black hole during the next orbit than at its present position. The only exception is the innermost orbit with $r_{\text {los }}=0$, where the cluster has passed beyond pericenter and will reach a closer pericenter position only a few pc from the SMBH after half a revolution. The bottom panel in Figure 10 displays the minimum and maximum velocity during the next orbit. Both the minimum GC distance and the maximum velocity show pronounced extrema at $r_{\text {los }}=20 \mathrm{pc}$, where the cluster follows an almost radial trajectory toward and away from the GC. The spike in the maximum velocity curve corresponds to the point of closest approach, where the cluster passes the black hole within a few parsecs.

The projected orbit into the immediate future of the cluster provides additional clues on the likelihood of cluster survival. Figure 11 shows the period of the next orbit. If the cluster presently resides in the inner $100 \mathrm{pc}$, it will have completed about one orbit in its lifetime (see also Fig. 8, bottom panels). At larger GC distances, the section of the orbit covered becomes accordingly smaller (see also Fig. 8, top panels). At line-of-sight distances $r_{\text {los }}>100 \mathrm{pc}$, the cluster remains at distances $r_{\mathrm{GC}}>100 \mathrm{pc}$ during the next orbit if it is presently located in front of the $\mathrm{GC}$, and at $r_{\mathrm{GC}}>65 \mathrm{pc}$ if it is located behind the $\mathrm{GC}$, suggesting that tidal forces will only intensely influence the cluster if it is found to be inside the inner $100 \mathrm{pc}$. This ambiguity shows the need for a precise distance measurement to the Arches cluster.

At a present-day line-of-sight distance of $-10 \mathrm{pc}$, the mean distance to the GC on the next orbit is about $70 \mathrm{pc}$, only marginally higher than the $62 \mathrm{pc}$ estimated from the circular orbit approximation. Nevertheless, the orbital period of 3.2 Myr is twice as long as the 1.6 Myr estimated for a circular orbit in a spherically symmetric potential from the parameters derived in $\S 4$, showing the need to account for a realistic potential in the inner Galaxy. At line-of-sight distances of $300 \mathrm{pc}$ and more, the cluster needs about 15 Myr to complete one orbit.

In summary, the Arches cluster has completed at most one orbit during its lifetime, and can pass through the inner $10 \mathrm{pc}$ only if the cluster's galactocentric radius is very close to its projected distance of $26 \mathrm{pc}$. These constraints imply that the Arches cluster cannot deposit young stars into the GC, supporting the prediction from $N$-body simulations by Kim \& Morris (2003) that the dense cores of clusters at large projected radii $\left(r_{\mathrm{GC}}>10 \mathrm{pc}\right)$ are unlikely to reach the central parsec even on highly elongated orbits. The orbital analysis incorporating the high orbital motion of the cluster thus provides the first observational evidence that Arches-like clusters are neither progenitors of the nuclear cluster, nor contribute significantly to the young population in the central parsec.

\subsection{Comparison with Dense Clouds in the $C M Z$}

As pointed out at the end of $\S 4.2$, the dense, massive, molecular clouds in the $\mathrm{CMZ}$ are found inside $200 \mathrm{pc}$ with moderate velocities of up to $120 \mathrm{~km} \mathrm{~s}^{-1}$. With knowledge of the cluster's 


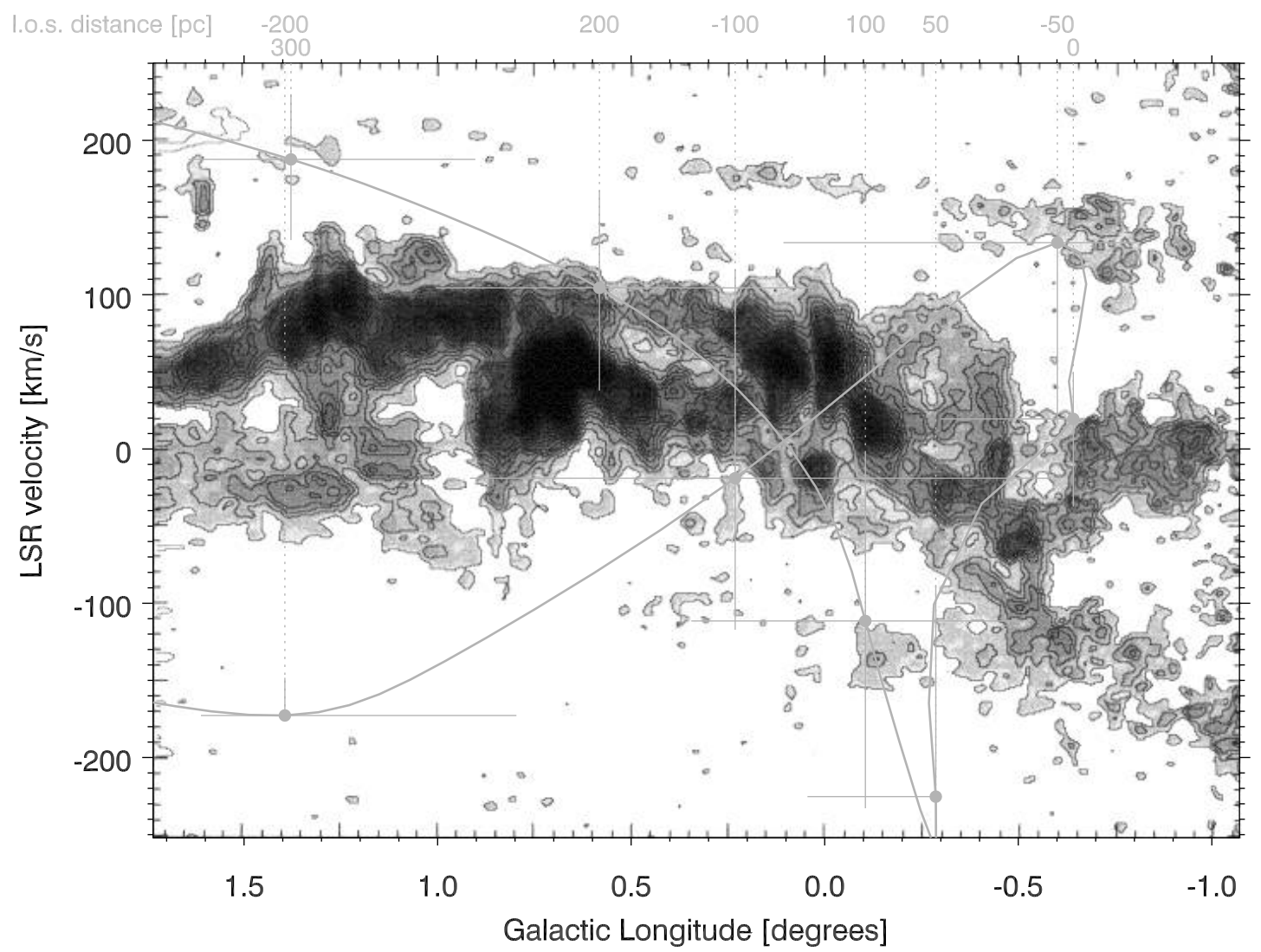

FIG. 12.-CS $J=1-0\left(l, v_{\text {los }}\right)$ map of dense molecular clouds in the CMZ as observed by Tsuboi et al. (1999). The position of the Arches integrated backward along its orbit for $2.5 \mathrm{Myr}$, is shown as blue dots, and the solid lines are error bars allowing for an age uncertainty of $\pm 0.5 \mathrm{Myr}$. The present-day line-of-sight distance of the cluster is labeled on the top axis, and the dotted lines connect each data point with the corresponding present-day line-of-sight distance. Initial conditions for line-ofsight distances $>200 \mathrm{pc}$ are indicative due to the neglected rotation of the bar. [See the electronic edition of the Journal for a color version of this figure.]

initial conditions, the $\left(l, v_{\text {los }}\right)$ location of the native clouds of the Arches at the time of its birth can be entered into the CS map of dense molecular gas observed in the GC today (Fig. 12, see Fig. 2 in Tsuboi et al. 1999). Error bars allow for an age uncertainty of $2.5 \pm 0.5$ Myr for the Arches (Najarro et al. 2004).

Assuming that the spatial distribution of clouds 2-3 Myr ago was similar to the CMZ observed today, ${ }^{10}$ the extrapolated initial conditions for the Arches cluster coincide with the location of dense, massive clouds $\left(M \sim 10^{6} M_{\odot}\right.$; Miyazaki \& Tsuboi 2000) for most of the line-of-sight distances. Large line-of-sight distances above $200 \mathrm{pc}$ in front of or behind the GC appear less likely because of the expected high initial velocities, $v_{\text {los }}>170 \mathrm{~km} \mathrm{~s}^{-1}$, significantly larger than the observed cloud velocities, $v_{\text {los }} \leq 120 \mathrm{~km} \mathrm{~s}^{-1}$. At present-day line-of-sight distances larger than $300 \mathrm{pc}$ from the $\mathrm{GC}$, the orbital analysis suggests that initial velocities would have to be $v_{\text {los }}>300 \mathrm{~km} \mathrm{~s}^{-1}$, which is unusually large for a starforming cloud in the CMZ. At small radii, the initial velocities depend strongly on the present age. In the inner $100 \mathrm{pc}$, essentially all initial velocities are possible, indicating that the initial velocity is not well constrained at small GC distances. In summary, the comparison with the space-velocity distribution of dense, massive clouds in the CMZ indicates a preferred formation locus inside the inner $200 \mathrm{pc}$ for the Arches. The possible orbital paths of the cluster suggest that the Arches is in this case also within \pm 200 pc of the GC today (see Fig. 9).

10 The maximum $x 2$ line-of-sight velocity does not change strongly with the rotation of the bar. See Binney et al. (1991) for effects of changes in the inclination angle on the $\left(l, v_{\text {los }}\right)$ diagram.

\section{SUMMARY AND CONCLUSIONS}

We measure the proper motion of the Arches cluster with respect to the surrounding field to be $212 \pm 29 \mathrm{~km} \mathrm{~s}^{-1}$. Combination with the line-of-sight velocity yields the $3 \mathrm{D}$ space motion of the cluster relative to bulge stars, $v_{3 \mathrm{D}}=232 \pm 30 \mathrm{~km} \mathrm{~s}^{-1}$. The high orbital velocity is inconsistent with a circular orbit in a spherically symmetric GC potential.

In order to constrain the orbit, we evolve the cluster in a more realistic potential following the observed mass distribution in the inner Galaxy. We obtain the cluster's initial position and velocity as a function of its present-day line-of-sight distance. Comparison with CS observations suggests that the initial position and line-of-sight velocity of the Arches coincide with x2 orbits of dense and massive $\left(\sim 10^{5}-10^{6} M_{\odot}\right.$; Miyazaki \& Tsuboi 2000$)$ molecular clouds if the cluster formed inside $r_{\mathrm{GC}}<200 \mathrm{pc}$, in which case it should still reside within 200 pc today. The high orbital velocity contradicts the recently suggested formation of the Arches as a rejuvenated globular cluster (Lin \& Murray 2007), as this scenario requires a small relative motion of the cluster with respect to $\mathrm{GC}$ clouds for an extended period of time.

In the asymmetric potential of the inner Galaxy, the Arches completed at most one orbit during its lifetime, and came into the central $10 \mathrm{pc}$ only if it is presently located closer than $33 \mathrm{pc}$ from the GC, close to its projected distance. These constraints restrict the amount of material possibly tidally stripped from the cluster, and render it highly unlikely that star clusters with Arches properties suffer sufficient energy loss to migrate inwards during the lifetime of the massive stars and contribute to the young population in the central parsec of the GC. This implies that with the 
Arches and Quintuplet clusters we are not observing progenitors to the GC comoving groups, providing observational confirmation of inspiral simulations (Gerhard 2001; Kim \& Morris 2003). This supports the origin of the nuclear cluster from a substantially more massive cluster at small radii a few million years ago, or via in situ star formation in the immediate GC environment.

Given the young age of the cluster and its long orbital relaxation timescale in the inner Galaxy, the large space velocity should be inherited from the natal cloud. When comparing the cluster orbital motion with line-of-sight velocities of gas clouds in the inner Galaxy using models of orbits in the dominating bar potential, we find that the $3 \mathrm{D}$ cluster motion is inconsistent with the closed $\mathrm{x} 1$ and $\mathrm{x} 2$ orbits suggested for gas clouds.

Although ample molecular material exists in the inner $200 \mathrm{pc}$ which could harbor the formation of clusters like the Arches, the dense, massive clouds have maximum line-of-sight velocities below $120 \mathrm{~km} \mathrm{~s}^{-1}$, consistent with $\times 2$ orbital velocities, but significantly lower than the present-day orbital velocity of the cluster. In the standard cloud fragmentation scenario, a dense, massive cloud must have existed on a high-velocity orbit $2.5 \mathrm{Myr}$ ago to generate the cluster. In addition, if a significant fraction of the dense clouds on $\mathrm{x} 2$ orbits would form starbursts, more clusters like the Arches or Quintuplet should be observed. The low number of clusters in comparison to clouds on $\mathrm{x} 2$ orbits and the high proper motion of the Arches indicate that the starburst event that could give rise to a massive compact cluster might have been triggered by a cloud-cloud collision.

Intense cloud-cluster interaction is observed from the X-ray bow shock of the Arches with its ambient medium by Wang et al. (2006), who suggest that a cloud-cloud collision could also have triggered the formation of the cluster. Following Binney et al. (1991), the starburst could have been triggered while the native cloud of the Arches collided on the boundary between $\mathrm{x} 1$ and $\mathrm{x} 2$ orbits in the inner bar, or from dispersed cloud material crossing the $x 1-x 2$ transition region. The backward integration of the cluster orbit is consistent with this formation locus if the cluster resides in the inner $200 \mathrm{pc}$. However, for the Arches to inherit the large orbital velocity from an $\mathrm{x} 1$ cloud, this model requires a massive, high-density cloud orbiting on an x1 orbit $2.5 \mathrm{Myr}$ ago, while clouds sufficiently dense to be triggered into an intense starburst event are only observed in the $\mathrm{x} 2$ orbital zone today. Alternatively, a collision with an infalling high-velocity cloud (e.g., Crawford et al. 2002) could also trigger star formation and propel the cluster on a highly eccentric orbit. The fact that the Arches moves predominantly parallel to the Galactic plane and has a vertical velocity of only $10 \mathrm{~km} \mathrm{~s}^{-1}$ indicates that the cluster did not originate from a collision with a vertically infalling cloud. If both the Arches (2.5 Myr ago) and Quintuplet (4 Myr ago), as well as Sgr B2 were triggered by cloud collisions, this would indicate a substantial cloud collision in the inner $\sim 200 \mathrm{pc}$ approximately every 2 Myr.

A cloud collision was suggested as the trigger for massive star formation in Sgr B2 by Hasegawa et al. (1994), who interpret the velocity structure observed in ${ }^{13} \mathrm{CO}$ as a dense clump colliding with a massive molecular cloud of lower density. These authors investigate two scenarios of cloud collisions near the Galactic center (1) a collision between two CMZ clouds, one of which moves on an elongated orbit in the bar, and (2) an infalling cloud originating above the Galactic plane. The first scenario can originate from a cloud on an x1 orbit moving into higher density clouds on $\mathrm{x} 2$ orbits, as suggested above. However, Hasegawa et al. (1994) crudely estimate the rate for such collisions to be as low as one collision per $200 \mathrm{Myr}$, which could not explain the occurrence of the Arches, Quintuplet, and Sgr B2 at the same time. These authors point out that the rate of infalling clouds is not well constrained, and they do not provide an estimate for the second scenario. Bally et al. (1988) identify a low-density molecular structure that is inclined by up to $40^{\circ}$ out of the Galactic plane, and point out that material on such inclined orbits has to cross the CMZ clouds in the plane twice along one orbital path, leaving ample opportunity for cloudcloud interactions. Alternatively, the Arches could have an exceptionally high velocity, and might have formed in a different mode than its neighbors. Currently, neither the collision rate nor the spontaneous starburst rate in the GC are well constrained. The observations of $3 \mathrm{D}$ space motions of the neighboring clusters will shed additional light on their origin.

Our measurement of the cluster motion will constrain dynamical evolution models in a realistic bar and nuclear cusp potential including energy loss along the orbit, which is the required next step to quantify the tidal mass loss of the cluster. Finally, the proper motion selection of cluster members provides us with a powerful tool to remove the field contamination, and derive the tidal structure and unbiased stellar mass function of the Arches cluster, which will be discussed in a forthcoming paper.

The authors wish to thank the anonymous referee for thoughtful comments on the manuscript and in particular on the cluster formation scenarios. We thank Brad Hansen, Mike Rich, and Hong Sheng Zhao for insights on the dynamical properties in the inner Galaxy and orbital considerations, and Ralf Launhardt for kindly providing his enclosed mass measurements and density distributions, and acknowledge fruitful discussions with Mike Muno and Claire Max. W. B. acknowledges support by a Julian Schwinger Fellowship at UCLA. This work would not have been possible without the intense effort and dedication of the Keck LGS-AO staff. We are deeply grateful for their support enabling these observations. The W. M. Keck Observatory is operated as a scientific partnership among the California Institute of Technology, the University of California, and the National Aeronautics and Space Administration. The Observatory was made possible by the generous financial support of the W. M. Keck Foundation. The authors wish to recognize and acknowledge the very significant cultural role and reverence that the summit of Mauna Kea has always had within the indigenous Hawaiian community. We are most fortunate to have the opportunity to conduct observations from this mountain. This work was supported by NSF grant AST 04 06-816 and by the Science and Technology Center for Adaptive Optics, managed by the University of California at Santa Cruz under cooperative agreement AST 98 76-783.

\section{REFERENCES}

Alves, D. R. 2000, ApJ, 539, 732

Athanassoula, E. 1989, Proceedings of the Joint Varenna-Abastumni Workshop on Plasma Phys., ed. T. D. Guyenne \& J. J. Hunt (ESA SP-285; Paris: ESA), 341

Bally, J., Stark, A. A., Wilson, R. W., \& Henkel, C. 1988, ApJ, 324, 223

Binney, J., \& Tremaine, S. 1987, Galactic Dynamics (Princeton: Princeton

Univ. Press), 214, 514

Binney, J., et al. 1991, MNRAS, 252, 210
Bissantz, N., Englmaier, P., \& Gerhard, O. 2003, MNRAS, 340, 949

Bissantz, N., \& Gerhard, O. 2002, MNRAS, 330, 591

Cotera, A. S., Erickson, E. F., \& Colgan, S. W. J. 1996, ApJ, 461, 750

Crawford, I. A., Lallement, R., Price, R. J., et al. 2002, MNRAS, 337, 720

Dame, T. M, Hartmann, D., \& Thadeus, P. 2001, ApJ, 547, 792

Diolaiti, E.; Bendinelli, O.; Bonaccini, D., et al. 2000, A\&AS, 147, 335

Englmaier, P., Gerhard, O 1999, MNRAS, 304, 512

Figer, D. F., Kim, S. S., Morris, M., et al. 1999, ApJ, 525, 750 
Figer, D. F., Najarro, F., Gilmore, D., et al. 2002, ApJ, 581, 258

Fruchter, A. S., \& Hook, R. N. 2002, PASP, 114, 144

Gerhard, O. 2001, ApJ, 546, L39

Ghez, A. M., et al. 2003, ApJ, 586, L127 2005, ApJ, 620, 744

Hasegawa, T., Fumio, S., Whiteoak, J. B., \& Miyawaki, R. 1994, ApJ, 429, L77

Kim, S. S., Figer, D. F., Lee, H. M., \& Morris, M. 2000, ApJ, 545, 301

Kim, S. S., \& Morris, M. 2003, ApJ, 597, 312

Kim, S. S., Morris, M., \& Lee, H. M. 1999, ApJ, 525, 228

Lang, C. C., Cyganowski, C., Goss, W. M., \& Zhao, J.-H. 2003, Astron. Nachr. Suppl., 324, 1

Launhardt, R., Zylka, R., \& Mezger, P. G. 2002, A\&A, 384, 112

Lejeune, T., \& Schaerer, D. 2001, A\&A, 366, 538

Lin, D. N. C., \& Murray, S. D. 2007, ApJ, 666, 779

Lindqvist, M, Winnberg, A., Habing, H. J., \& Matthews, H. E. 1992, A\&A, 92, 43
Miyazaki, A., \& Tsuboi, M. 2000, ApJ, 536, 357

Morris, M. 1993, ApJ, 408, 496

Nagata, T., Woodward, C. E., Shure, M., \& Kobayashi, N. 1995, AJ, 109, 1676 Najarro, F., Figer, D. F., Hillier, D. J., \& Kudritzki, R. P. 2004, ApJ, 611, L105 Portegies Zwart, S. F., Makino, J., McMillan, S. L., \& Hut, P. 2002, ApJ, 565, 265 Rattenbury, N. J., Mao, S., Sumi, T., \& Smith, M. C. 2007, MNRAS, 378, 1064 Schoedel, R., Eckhard, A., Alexander, T., et al. 2007, A\&A, 469, 125

Stolte, A., Brandner, W., Grebel, E. K., Lenzen, R., \& Lagrange, A.-M. 2005, ApJ, 628, L113

Stolte, A., Grebel, E. K., Brandner, W., \& Figer, D. F. 2002, A\&A, 394, 459 Sumi, T., Eyer, L., \& Woźniak, P. R. 2003, MNRAS, 340, 1346

Tsuboi, M., Handa, T., \& Ukita, N. 1999, ApJS, 120, 1

van Langevelde, H. J., et al. 1992, A\&A, 261, L17

Wang, D. Q., Dong, H., \& Lang, C. 2006, MNRAS, 371, 38 